DOE Grant Number DE-FG02-04ER41323 to Northern Illinois University

Title:

\title{
Nonlinear Dynamics of High-Brightness Electron Beams and Beam- Plasma Interactions: Theories, Simulations, and Experiments
}

Principal Points of Contact:

Technical: Prof. Philippe Piot

Northern Illinois University

Department of Physics

DeKalb, IL 60115

Phone: (815) 753-6473; Fax (815) 753-8565;

Email:piot@nicadd.niu.edu

Administrative: Dean James Erman

Northern Illinois University

Office of Sponsored Projects

DeKalb, IL 60115

Phone: (815) 753-9282; Fax : (815) 753-1631

Email: asosp@niu.edu

DOE Program Manager: Dr. L. K. Len/Dr. W. Weng

SC-25/Germantown Building

U.S. Department of Energy

1000 Independence Avenue, SW

Washington, D.C. 20585-1290

Phone: (301) 903-3233; Fax: (301) 903-2597

Email: L.K.Len@science.doe.gov

Date: 31 May 2008 


\section{Project Overview}

According to its original Statement of Work (SOW), the overarching objective of this project is: "To enhance substantially the understanding of the fundamental dynamics of nonequilibrium high-brightness beams with space charge." Our work and results over the past three and half years have been both intense and fruitful. Inasmuch as this project is inextricably linked to a larger, growing research program - that of the Beam Physics and Astrophysics Group (BPAG) - the progress that it has made possible cannot easily be separated from the global picture. Thus, this summary report includes major sections on 'global' developments and on those that can be regarded as specific to this project.

\section{Students}

This grant supported, in full or in part, five graduate students:

- Mr. Marwan Rihaoui, who is doing research on beam physics in photoinjectors with a focus on space charge and its impact on beam dynamics, and is working at Argonne's Advanced Wakefield Accelerator Facility. Mr. Rihaoui passed his Ph.D. candidacy exam in February 2006 and is now a Ph.D. candidate.

- Mr. Edward Nissen, who is doing research concerning the onset and influence of chaotic orbits in beams with space charge. Ed passed both his MS and PhD physics candidacy exams in his first year of matriculation at NIU, which is unprecedented for our Department. He also won a $10 \mathrm{k} \$$ fellowship from the Directed Energy Professional Society that has partially compensated his graduate-research-assistant (GRA) salary. For his MS thesis, Ed is presently working in the context of FFAG synchrotron designs, and has been getting input from S. Y. Lee of the University of Indiana, as well as Giuliano Franchetti from GSI, Darmstadt, Germany. He then started his $\mathrm{PhD}$ work on the University of Maryland Electron Ring.

- Mr. Timothy Maxwell, who is working as a laser physicist in BPAG's new Laser Laboratory. Tim is operating our new femtosecond-class Ti:Saph laser and a relatively high-energy ultraviolet laser. The former is to be (initially) used to develop a new electro-optical (EO) beam diagnostic, and the latter is to be used as a photocathode drive laser in conjunction with a compact low-energy, high-brightness electron source we are developing in collaboration with Argonne National Laboratory. Tim's MS thesis will concern one or both of these areas. He has yet to pass his physics $\mathrm{PhD}$ candidacy exam, but he is an excellent student and will likely do so. He won twice a $5 \mathrm{k} \$$ NIU Fellowship that partially compensates his GRA salary.

- Ms. Shafaq Moten worked on technology associated with (1) developing a beam diagnostics capable of resolving the expected micron-sized electron beam produced by the aforementioned electron source, and (2) designing the ultraviolet optical beam line needed to photoemit electron bunches using the ultraviolet laser. This work consitsted the essential of Ms Moten's MS thesis. Ms. Moten was a "terminal" MS student and left NIU in August 2007.

- Mr. Greg Betzel, completed his MS in summer 2005 working on impact of chaos on emittance dilution. Mr. Betzel never did pass his Ph.D. candidacy exam. He worked for a few months as a technician for BPAG, during which time he was supported from other funds. Presently he is a graduate student at the University of Canterbury, New Zealand, where he is pursuing a Ph.D. in medical physics. 


\section{Faculty and Postdoctoral Research Associates}

Most of the research work (from the beginning of this grant to January 2007) was performed under the late Professor Court Bohn. Philippe Piot directed the research work starting January 2007 Philippe Piot supervised the thesis work of Marwan Riahoui and Shafaq Motem. Prof. Bela Erdelyi supervises the PhD work of Edward Nissen which started in September 2007. Several reseach associates sponsored, via other funds, contributed to this work. Dr. Sideris and Terzic respectively develop a phase space analysis technique and a space charge beam dynamics algorthm Dr. Mihalcea contributed to diagnostic development.

\section{Collaborations}

We continue our collaboration with the UMER Group at the University of Maryland, and the Principal Investigator (C. Bohn) now chairs the recently-formed UMER Advisory Committee. We have granted the UMER Group time to run WARP simulations on our 112-CPU Beowulf computer cluster, and have been applying our computational tools for measuring chaos to particle orbits from UMER simulations. We also continue our collaboration with Rob Ryne's Accelerator Modeling \& Advanced Computing Group at LBNL in regard to the development of accurate and efficient multiscale computational techniques. In fact, we will include them as a partner in the proposal that we will submit to ONR in early CY07. With one of Rob's staff, Dr. Ilya Pogorelov, we wrote two peerreviewed papers $[1,2]$. We also continue our collaboration with Fermilab personnel regarding the photoinjector and beam diagnostics for ILCTA. In fact, Prof. Philippe Piot, a member of BPAG, designed an RF photoinjector for ILCTA. He also recently cochaired in November 2006 a mini-workshop on possible advanced accelerator R\&D directions at the ILC Test Accelerator that Fermilab is thinking of building.

We began a new collaboration, this being with the Argonne Wakefield Accelerator (AWA) Group, first to do space-charge experiments with the AWA beam, then to do tests of beam diagnostics as well as experimental test of phase-space manipulations involving two degrees of.freedom. Space-charge experiments have begun in earnest during January 2007, coinciding with our detailing our student Mr. Marwan Rihaoui to work full-time at AWA..

We also collaborated with Prof. Uwe Happek at the University of Georgia toward the development, characterization, and use of an interferometric diagnostic for measuring the longitudinal charge density of bunched beams.

\section{Summary of achievements}

What follows is a report that is organized per the specific tasks (listed in italics) delineated in the Statement of Work.

1. Ascertain the relationship between the time-dependent potential of a nonequilibrium beam and the population of globally chaotic orbits that the beam supports. 
We performed a systematic study of a 'simple' family of 1D time-dependent models corresponding to breathing spherically symmetric waterbag potentials [Bohn, et al., NI M A561, 230 (2006)]. There are several attractive advantages of the spherical model: (1) the distribution function of particles in the phase space of a single particle, the chargedensity profile, and the space-charge potential are all analytic; (2) the space-chargedepressed tune $\eta$ parameterizes the family of models, so one can readily explore the range from zero space charge to the space-charge limit; (3) the equilibrium configurations are all stable, and (4) whereas all of the equilibrium configurations are integrable in keeping with spherical symmetry, a range of models admits chaotic orbits once they are made to breathe. Hence, when it is present, this deterministic chaos is fully attributable to the time dependence alone. Thus, this family of models is ideal not only for answering basic questions such as how time dependence triggers chaoticity, but also for highlighting the efficacy of new techniques for quantifying this chaos and for analyzing nonlinear dynamics in general. From Fig.1, we see that this model generates a sizeable population of chaotic orbits near its boundary. Halo subsequently develops as a consequence of this chaos, as seen in Fig. 2.

We also discovered evidence that chaos is not required to form a halo. Specifically, we simulated the beam dynamics in a photoinjector, known as the 'AES/JLab Photoinjector', that is presently under construction at Jefferson Lab. We found that, in generating 1-nC bunch charges, if the cathode spot is smaller than about $2.8 \mathrm{~mm}$ in radius, then copious halo is formed. This machine is short; there is simply not enough time for the constituent electrons to execute more than a single 'betatron' oscillation, and this time frame is too short for orbits to mix appreciably. Instead, as a consequence of space charge alone, a fraction of the beam is launched to large amplitudes by a phenomenon akin to 'tidal shocking'. This mechanism is illustrated in Figs. 3 and 4.

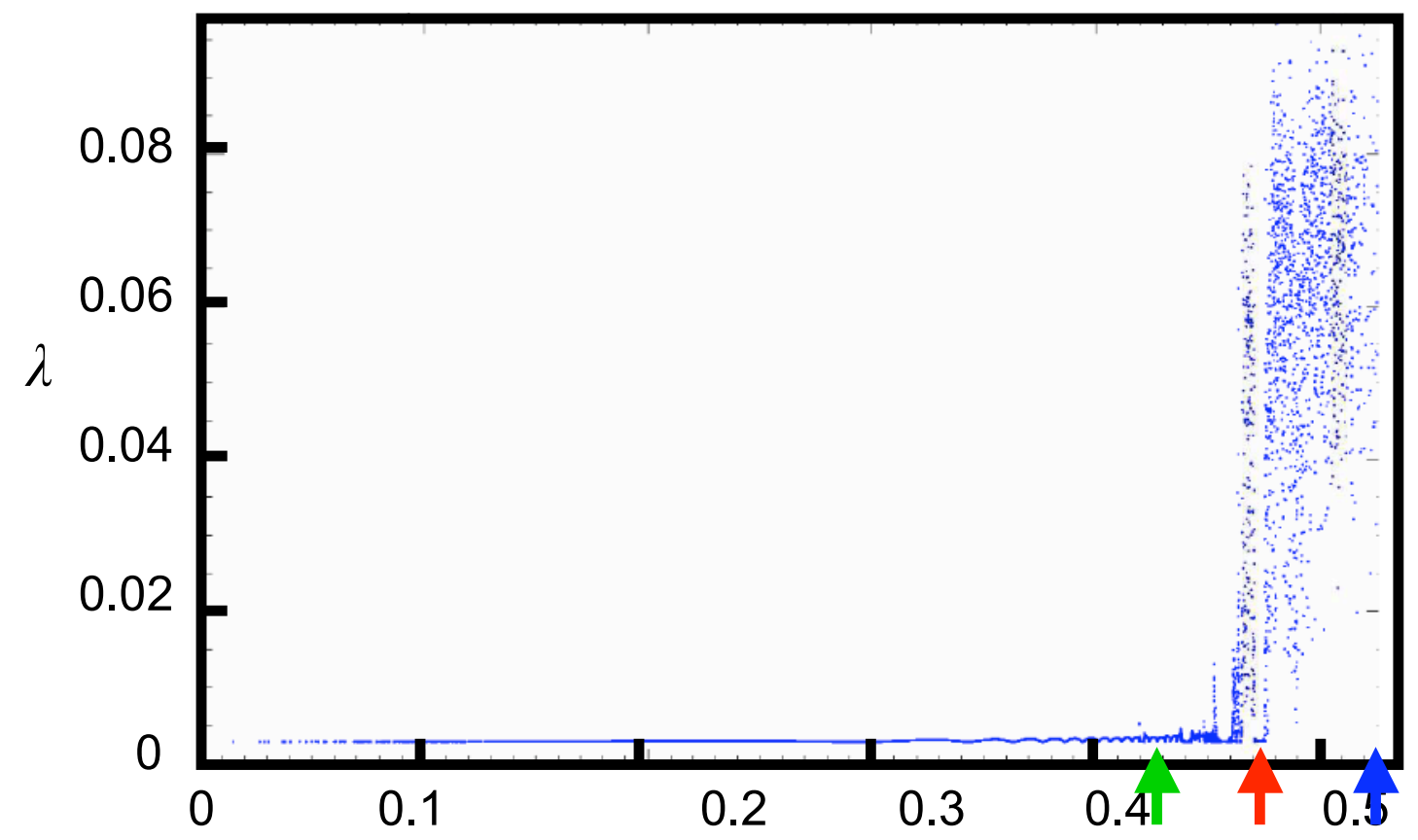


Figure 1. Standard Lyapunov exponents $\lambda$ of 10,000 orbits vs. initial condition $x_{0}\left(x^{\prime}(0)=0\right.$ for every orbit), with tune $\eta=0.15$ and mismatch $M=0.50$. Three clumps used for mixing experiments with regular orbits (green), 'sticky' chaotic orbits (red), and wildly chaotic orbits (blue) were centered at $x_{0}=0.425,0.475$, and 0.525 , respectively, the latter corresponding to just inside the boundary of the waterbag at $t=0$.

2. Ascertain how the globally chaotic orbits distribute themselves through their accessible phase space. What is/are the time scale(s), and how does it(they) depend on the beam parameters? What macroscopic structure ensues within the beam as a result? What is the quasi-static end product of the associated phase mixing?

Again, within the context of a simplified model, Fig. 2 largely answers these questions at a fundamental level. Of course, a perpetually breathing waterbag never settles to a static state, and so it provides no information regarding the "end product".

What is utterly fascinating is that one can 'watch' clumps of test particles that are initially tightly localized in phase space evolve to fill in the Poincare surface of section (PSS); the wildly chaotic orbits quickly migrate to fill the chaotic sea, 'sticky' chaotic orbits eventually break into the chaotic sea and migrate away, while the regular orbits slowly mix to fill the regular islands. One can likewise watch chaotic particles migrate to form a halo. In fact, chaotic orbits mix exponentially through the phase space accessible to them, while regular orbits mix secularly (as a power law in time); these qualitative differences are manifest in Fig. 5.
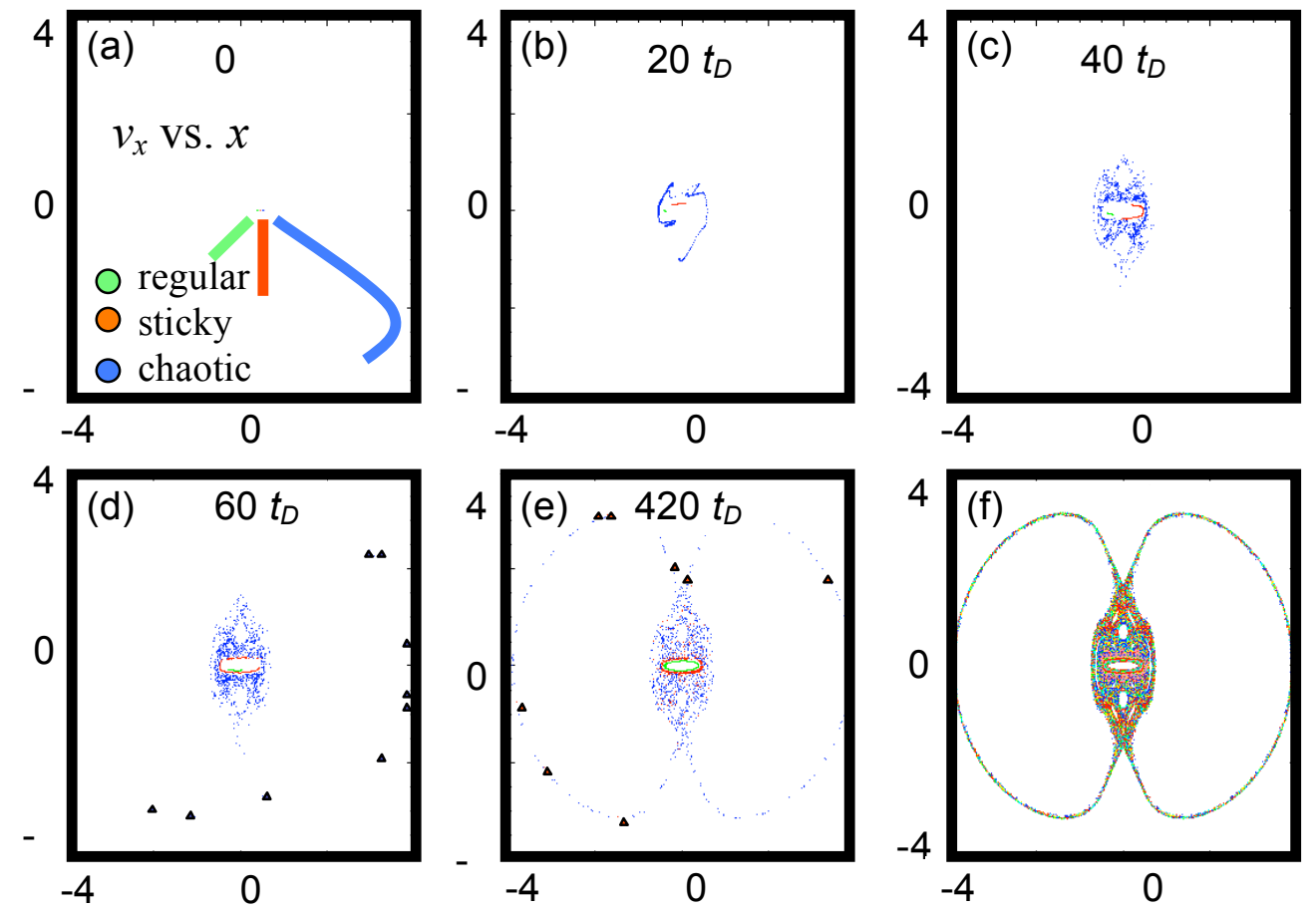

Figure 2. Stroboscopic Poincaré sections for the waterbag of Fig. 1. Orbital coordinates are plotted when the waterbag has its minimum radius. Plots (a-e) show evolution of three clumps, each with 1,000 test particles. In (d), triangles flag newly born halo particles. In (e), triangles flag red particles that became 'unstuck' from their regular islands, migrated through the chaotic sea, coupled to the 2:1 resonance, and shot into the halo. Plot (f) shows the complete section computed with 500 test particles initially distributed per the waterbag density profile (the color coding here is arbitrary). 

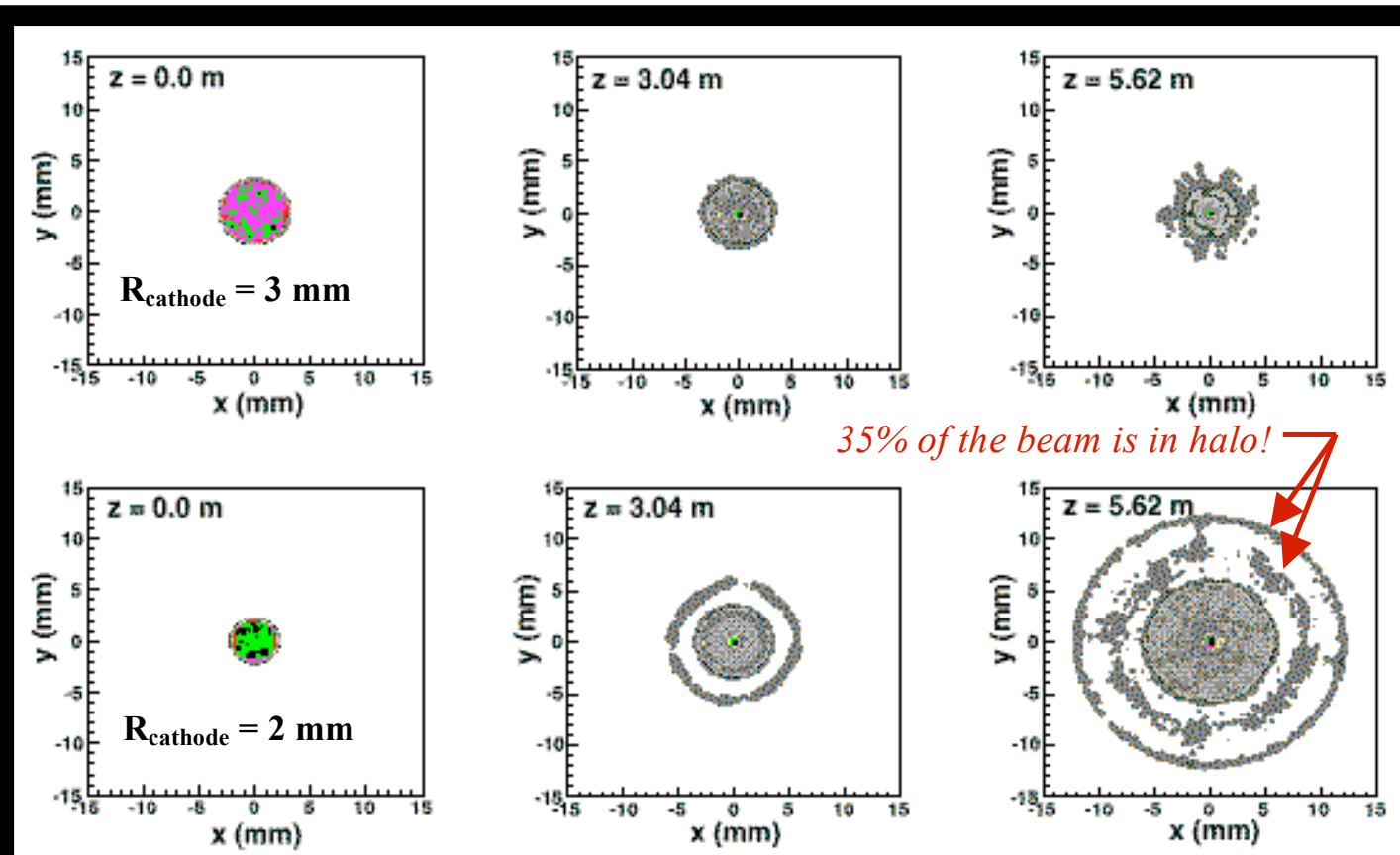

Figure 3. Transverse halo in 1-nC bunches generated with the AES/JLab Photoinjector and given a cathode spot of radius $3 \mathrm{~mm}$ (ton) and $2 \mathrm{~mm}$ (hottom). The rightmost nanels corresnond to the output beam.

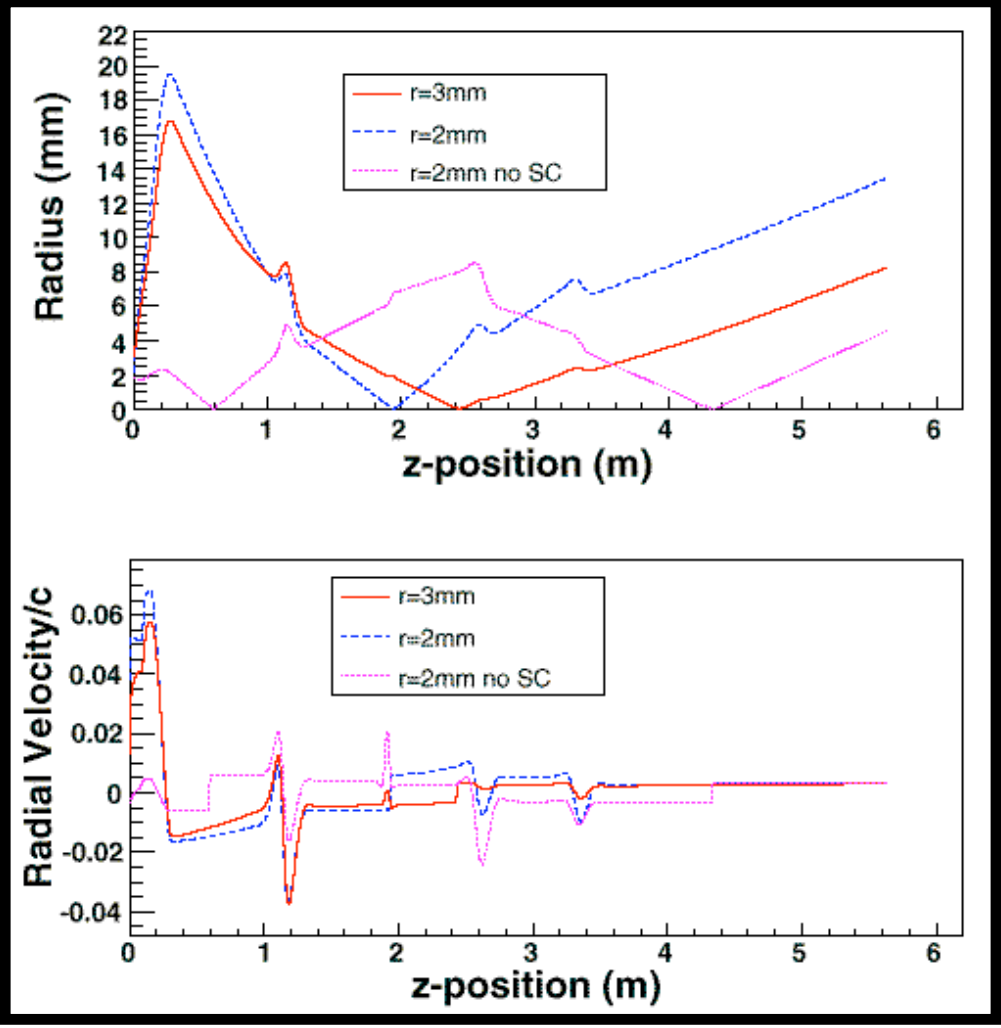

Figure 4. Orbit of the outermost particle at the exit of the AES/JLab Photoinjector. Note the launching to large amplitude at early time. Also note that if space charge is turned off, there is no such launching. 


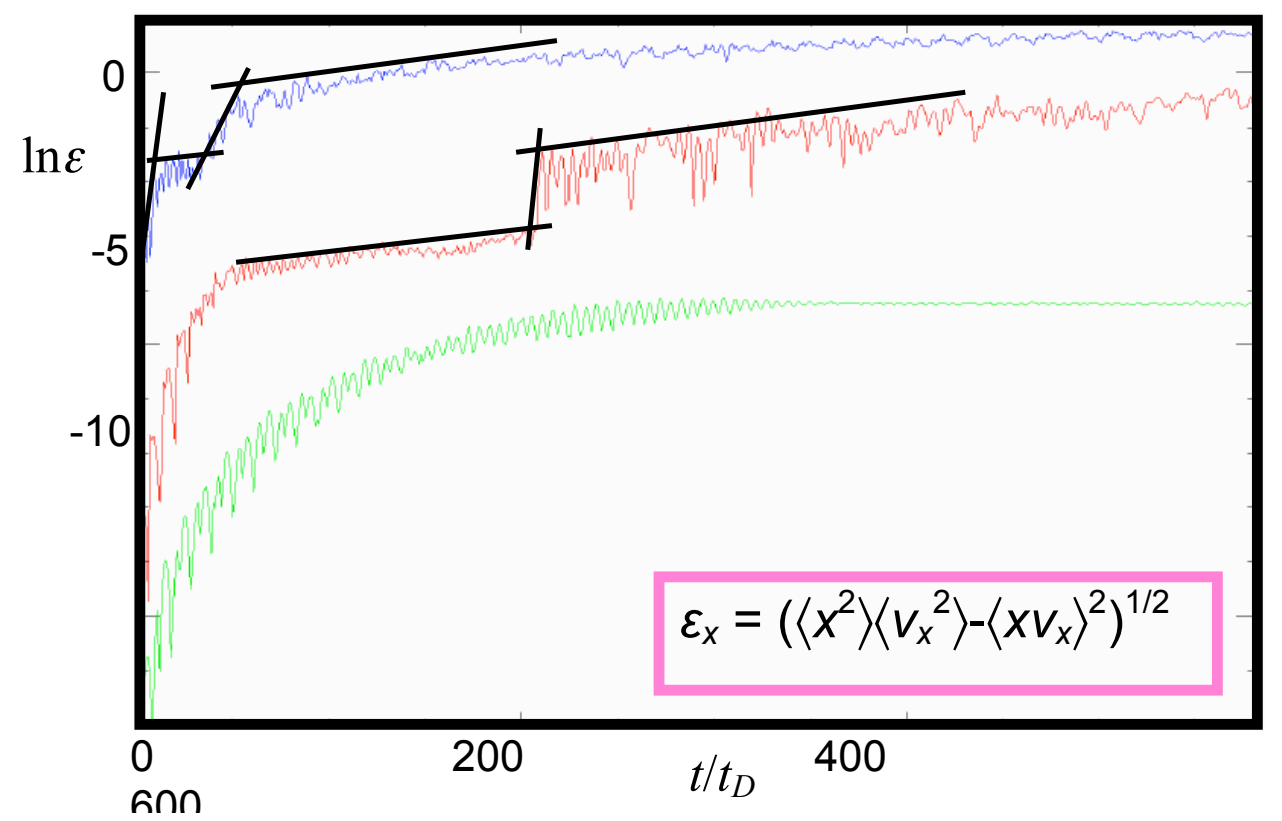

Figure 5. Plot of the logarithm of the clump emittance $\varepsilon_{x}$ vs. $t$ for the three clumps of Fig. 2 . The lines signify exponential scaling of the emittance with time. Note that the emittance of the regular (green) clump grows as a power-law in time, not exponentially. Jumps occur where particles begin to enter the halo.

3. Ascertain how particle energies redistribute themselves in response to a timedependent potential. Does energy redistribution proceed qualitatively in the same way as phase-space redistribution?

We also investigated this topic using the breathing spherical waterbag [G. Betzel, MS Thesis (2005)]. Because these models are so simple, one can track the mixing of energies just as readily as one can track the mixing of orbits through phase space. The evolution of the energy spread in the three clumps of Fig. 2 appears in Fig. 6. One sees that for this model the energy redistribution does behave in a qualitatively similar way as the respective emittance growth. This seems to indicate that the initial energy redistribution is exponential rather than diffusive (power-law); however, investigations with more realistic models are needed to support a general conclusion.

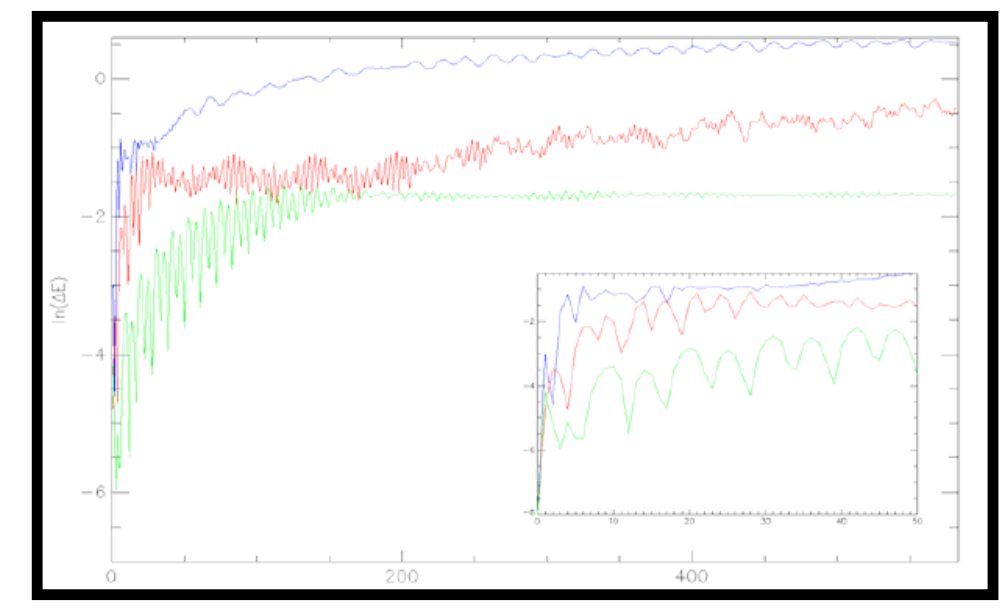


Figure 6. Natural logarithm of the root-mean-square energy spread $\ln (|\Delta E|)$ vs. $t_{D} \in[0,583]$ for the three clumps of Fig 2. The smaller panel helps illustrate initial exponential or power law growth.

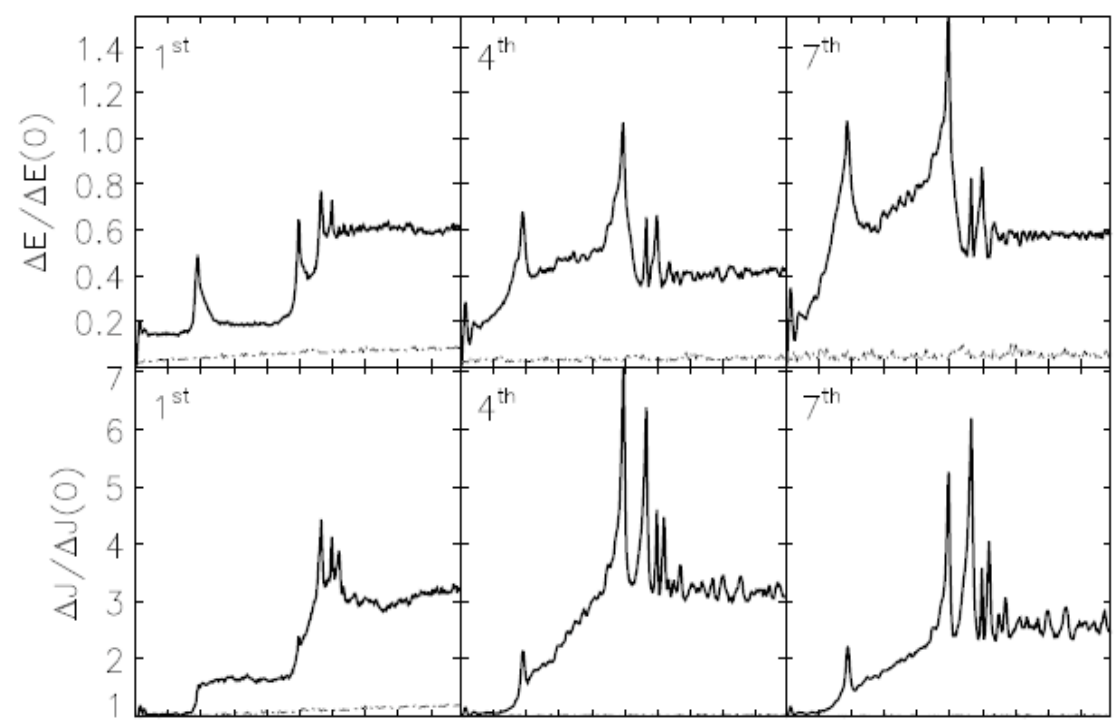

012345678901234567890123456789

Figure 7. Spread of energy (top) and angular momentum (bottom) vs. time for three different shells in the dark-matter halo. The solid curves are for the merger of two halos, while the dot-dashed curves are for an isolated halo that was used as a control in this numerical experiment.

We also explored this phenomenology in a gravitational analogue, to wit, the merging of two dark-matter Navarro-Frenk-White halos [Valluri, et al., ApJ. (in press)]. Initially these objects are spherically symmetric, and they collide nearly head-on. During the merger the system oscillates violently, but only for a few (about 4) periods, after which the merger product executes only small-amplitude oscillation. Here, a halo is formed, and the redistribution of particles is found to be due to tidal shocking. This is analogous to what happens in the AES/JLab Photoinjector; there simply is not enough time for particles to mix otherwise during the violent phase. The corresponding behavior of the energy spread (and angular-momentum spread) appears in Fig. 7. There one sees that the energy spread exhibits spikes; it does not evolve in any quasi-smooth manner at all. These spikes occur when the halo centroids pass near each other. The end result is that particles are "flung out"; they are redistributed due to gravitational tidal shocking.

4. Ascertain how granularity influences the evolution of nonequilibrium beams. What observable consequences might arise? Under what conditions might it be safe to invoke a coarse-grained (Vlasov-Poisson) representation of phase space, without or with a Fokker-Planck model of the noise associated with granularity?

Preserving a hierarchy of scales in the time-dependent space-charge potential is dynamically important. Recent research has revealed that nonlinear, time-dependent forces commonly establish large populations of globally chaotic orbits in beams that are out of equilibrium, and such orbits can even be present in thermal-equilibrium beams [Bohn \& Sideris, PRST-AB 6, 034203 (2003)]. When present, these chaotic orbits mix exponentially throughout their accessible phase space with a time scale of only a few orbital periods, i.e., very much faster than via collisional relaxation. The presence of colored noise due to space-charge fluctuations and/or machine imperfections can, when 
combined with parametric resonance associated with low-order oscillatory modes, generate large halos over just a few space-charge-depressed betatron periods. Thus, all scales are potentially important to the dynamics.

Multiresolution analysis is a mathematical discipline designed to preserve the physically important scales. It hinges on the use of 'wavelets', which are mathematical entities that are defined over a finite range (i.e., they have the property of 'compact support'). Wavelets are also scaleable; a superposition of wavelets distributed over a hierarchy of scales will reproduce very complicated structure, even with a relatively few number of wavelet coefficients. In view of these considerations, we developed an algorithm to solve Poisson's equation linking the charge density in a beam bunch to the space-charge potential. The use of wavelets generates potential-density pairs that correctly preserve the hierarchy of scales in the beam, thereby enabling accurate computations that apply well beyond predictions of conventional rms beam properties [B. Terzić, et al., PRST-AB (submitted)].

To be accurate, algorithms for solving Poisson's equation must:

1. include multiscale dynamics, because even fluctuations on smallest scales can lead to global instabilities, as exemplified by halo formation and microbunching [Bohn \& Sideris, PRL 91, 264801];

2. minimize numerical noise due to: (1) the number of macroparticles $N$ used to sample the phase-space distribution function in the $N$-body simulation being several orders of magnitude smaller than the number of real particles in the physical system that is being modeled (graininess of the distribution function), and (2) the continuous physical problem being restricted to a discrete, finite computational grid (discreteness of the computational domain); and

3. be efficient in terms of computational speed and storage requirements, without compromising accuracy.

With multiresolution analysis (wavelets), all three features are attainable, and these considerations contributed to our motivation to develop the wavelet-based Poisson solver. We implemented the new solver by plugging it into IMPACT-T, which was developed at Lawrence Berkeley National Laboratory for modeling accelerators of high-brightness beams [Qiang, et al., J. Comp. Phys. 163, 434 (2000); Qiang \& Ryne, Comp. Phys. Comm. 138, 18(2001)]. The 'standard' version of this code employs a Green function and fast Fourier transform to solve Poisson's equation.

After transforming the noisy charge distribution into wavelet space, the signal (physical component) is generally represented by a smaller number of large coefficients, while the numerical noise is largely mapped to many small wavelet coefficients. Wavelet thresholding is a process whereby the contribution of the wavelet coefficients deemed to represent noise is eliminated. Simply put, the wavelet coefficients whose magnitudes are below a certain noise threshold $T$ are set to zero. One must choose the noise threshold carefully, avoiding two extreme perils: on one end, over-smoothing the distribution by overestimating the threshold, and on the other end, not removing enough unphysical small-scale noise by underestimating the threshold. 
Studies of wavelet denoising usually concern distributions contaminated with additive (distribution-independent) Gaussian (white) noise. The most widely used noise threshold is given in terms of the standard deviation $\sigma$ of the noise as $T=\left(2 \log N_{g}\right)^{1 / 2} \sigma$, where $N_{g}$ is the total number of grid points. This is a universal threshold for signals with Gaussian noise, which means it leads to noise removal that is within a small factor of ideal denoising. However, noise in PIC simulations is Poisson-distributed and distributiondependent. The basic assumption underlying denoising techniques is that, regardless of the details of the noise, small-scale fluctuations due to noise map to small-scale members of the wavelet family. That said, one can transform a Poisson-distributed signal $X_{P}$ to a Gaussian-distributed signal $X_{G}$ with unit variance and mean using a variance-stabilizing transformation due to Anscombe: $X_{G}=2\left[X_{P}+(3 / 8)\right]^{1 / 2}$. Applying this 'Anscombe transform' produces a bias in the data; however, it can be removed by ensuring the denoised and noisy data have the same mean (in simulations, this is equivalent to enforcing charge conservation). When the number of particles per cell in the PIC simulation is too low, the noise exhibits a departure from the Poisson distribution, in which case the Anscombe transform breaks down. Typically one needs at least three particles per cell in the simulation to avoid this difficulty.

To verify a new code means to compare its output to that of established codes. The idea is to ensure the new code correctly incorporates the physics (here, the concern is spacecharge physics) while pinpointing differences in code predictions. We do so by comparing the detailed predictions concerning the beam's evolution in (1) the threedimensional configuration space alone, and (2) the full six-dimensional phase space. Here, we concentrate on simulating the Fermilab/NICADD photoinjector [http://www.nicadd.niu.edu/fnpl]. This machine includes a 1.625-cell radiofrequency (rf) electron gun enveloped by three solenoids for emittance compensation, followed by a nine-cell superconducting rf booster cavity, a 'chicane' of four dipole magnets for bunch compression, and a transport line composed of quadrupole magnets. The beam kinetic energy out of the gun is $\sim 4 \mathrm{MeV}$, and out of the cavity is $\sim 15 \mathrm{MeV}$.

Space charge forces and external fields dictate the beam dynamics. Since external fields are treated the same in all versions of IMPACT-T, differences in the beam dynamics may arise only from differences in how the space-charge force is computed. To verify agreement between the space-charge computation of the two codes, we tested them on two highly nonuniform transverse initial distributions of $1 \mathrm{nC}$ bunches: (1) a considerably nonuniform and asymmetric distribution generated from a real laboratory snapshot of the laser-illuminated photocathode in an actual experiment under suboptimal conditions (henceforth called 'Distribution 1'); and (2) a 5-beamlet quincunx distribution that can be made by masking the photocathode (henceforth called 'Distribution 2'). We expect that the nonuniformity and asymmetry of the two initial transverse distributions will strongly enhance space-charge effects vis-à-vis a uniform distribution, thereby 'stressing' the Poisson solvers. We compare results computed from these two distributions regarding: (1) rms properties of the beam, (2) phase-space detail, and (3) computational speed.

Figure 8 shows the rms properties of the beam in the Fermilab/NICADD photoinjector for Distribution 1 computed with conventional IMPACT-T (black lines), and with 
IMPACT-T with PCG without thresholding (green line), with thresholded after Anscombe transform (blue line), and with thresholding but without Anscombe transform (red line). Figure 9 shows the same for Distribution 2. Agreement of rms properties between the versions of IMPACT-T is excellent, to within a few percent.

For Distribution 1, the beam size in the laboratory experiment was measured at different positions along the beam line. Figure 6 compares the measurements against values computed using conventional IMPACT-T (red line) and IMPACT-T with PCG (blue line). This represents a first step toward code validation. Taken together, these results clearly demonstrate that simulations using both versions of IMPACT-T are in excellent agreement regarding the computation of beam moments. They also match the measured rms beam sizes reasonably well. We have also done some validation against the longitudinal charge distribution; details are documented elsewhere [20]. Regarding this work, both PARMELA and conventional IMPACT-T were the codes being validated; insofar as we have verified our wavelet version of IMPACT-T against the conventional one, we have also indirectly validated it.

For Distribution 2, Fig. 10 shows projected transverse cross-sections of the beam at different positions down the beamline. Detailed agreement between the configuration spaces computed with the two versions is clearly very good.
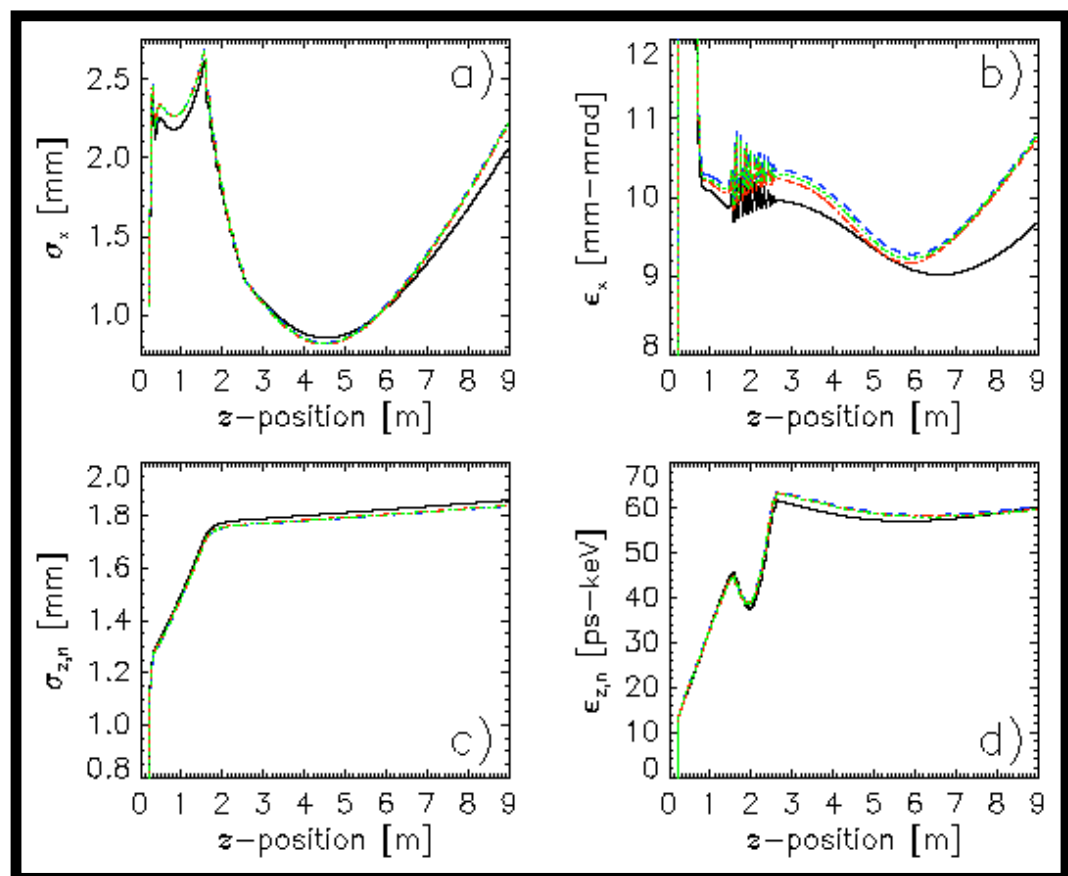

Figure 8. For Distribution 1: Simulation results for the Fermilab/NICADD photoinjector using $32^{3}$ grid points and 200,000 macroparticles. Curves correspond to: conventional version of IMPACT-T (black), IMPACT-T with PCG without denoising (green), IMPACT-T with PCG with thresholding and Anscombe transformation (blue), and IMPACT-T with PCG with thresholding but without Anscombe transformation (red). Computed quantities are: (a) rms beam radius, (b) rms normalized transverse emittance, (c) rms bunch length, (d) rms normalized longitudinal emittance. For IMPACT-T with PCG, we use Daubechies wavelets of order 2 . 


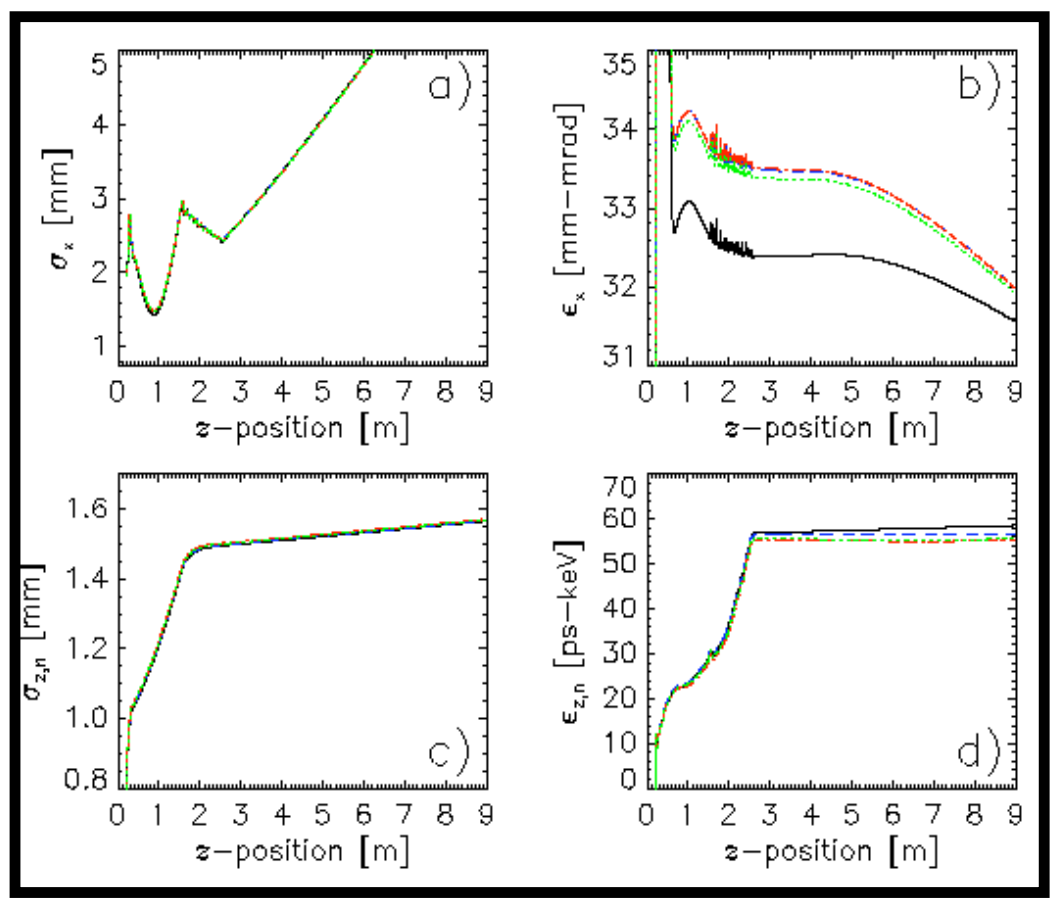

Figure 9. Same as in Fig.8, but for Distribution 2. 

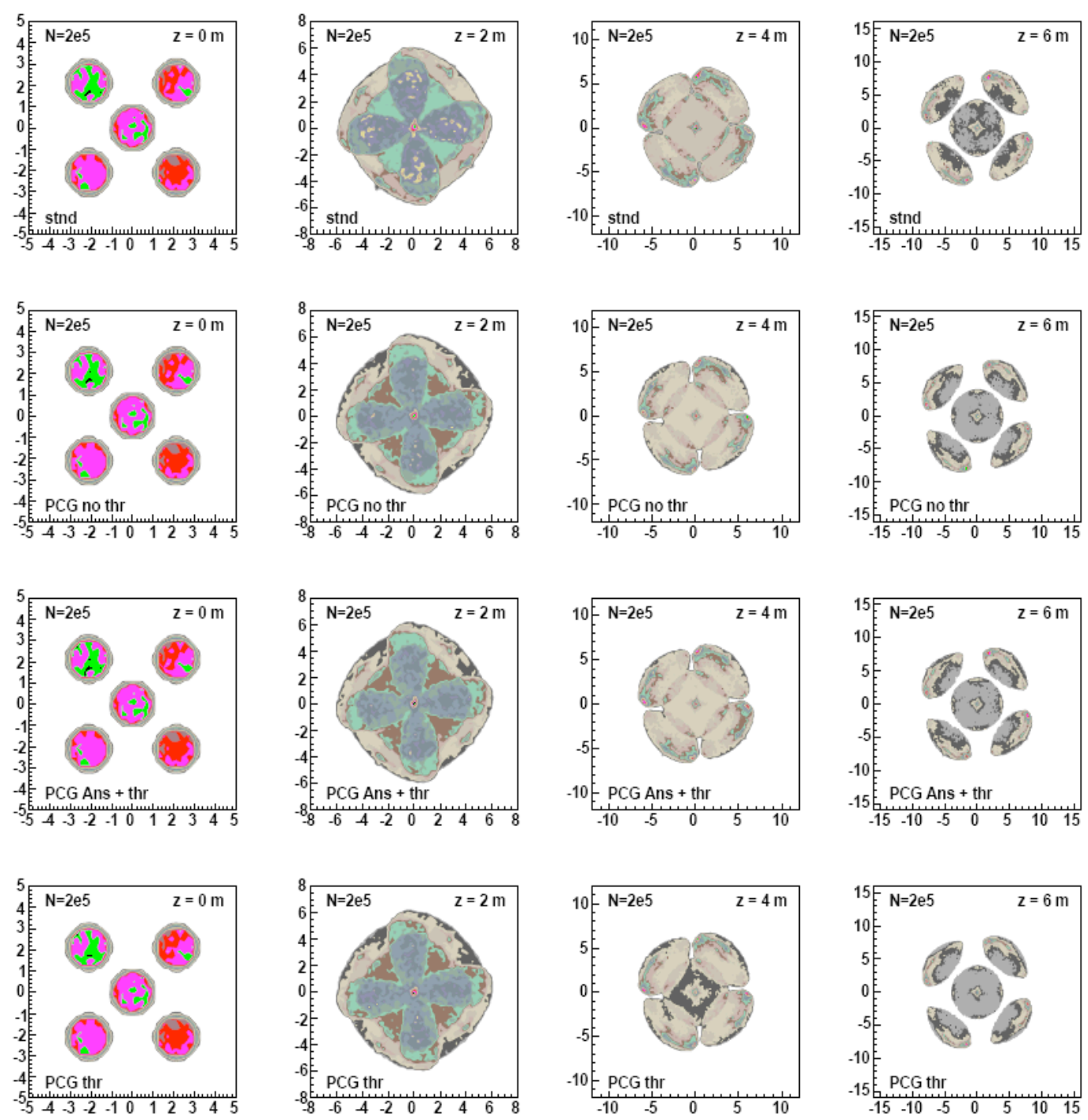

Figure 10. For Distribution 2: Integrated transverse cross-section of the beam at different positions along the Fermilab/NICADD photoinjector computed with $32^{3}$ grid points, 200,000 macroparticles, and Daubechies wavelets of order 2: conventional IMPACT-T (first row), IMPACT-T with PCG and no thresholding (second row), IMPACT-T with PCG and Anscombe transform and thresholding (third row), IMPACT-T with PCG and thresholding but no Anscombe transformation (fourth row). First column shows the beam leaving the cathode, second at $z=2 \mathrm{~m}$, third at $z=4 \mathrm{~m}$, and fourth at $z=6 \mathrm{~m}$.

To compare codes in the six-dimensional phase space, we invented a new technique for quantifying chaoticity in particle orbits. There have always been two main concerns related to chaotic measures: (1) the accuracy of the characterization, and (2) the speed, i.e. the length of time one must evolve an orbit to get a reliable characterization. For time-independent regimes (where energy is conserved, so regular orbits remain always regular, and chaotic orbits remain always chaotic), the longer the evolution time, the more accurate the characterization. However, the shortest evolution time required for confident characterization depends on the measure employed. The traditional Lyapunov exponents may need hundreds, or even thousands, of orbital periods to converge. The most sophisticated measures today have ostensibly lowered this limit to about 30 orbital 
periods, but this may depend significantly on both the chosen model and the specific orbit.

With these considerations in mind, we developed a new measure based on pattern recognition. It treats a signal (e.g., an orbit) not as one entity but as a series of distinct epochs. In this sense, it focuses on local, epochal characterization, instead of the usual approach of global characterization. Pictures of the phase space can be made, including essential details, typically with as little as just 10 orbital periods. Moreover, this measure applies to both time-independent and time-dependent systems without any change in its logic or design.

Perhaps the most attractive feature of our method is that it enables one to paint detailed phase-space portraits and make movies of the beam as it evolves in phase space. Thus, by comparing movies made from the output of different codes, one accomplishes detailed code verification. This is a much more sensitive verification than can be achieved by comparing computed rms properties (like beam moments) alone. A second very attractive feature is that, by making such movies, one can identify and follow the evolution of instabilities, particularly those that start on a localized scale and grow to macroscopic scales. In other words, it serves as a 'phase-space microscope'.

What we did for a first application toward code verification was to 'install' an artificial periodic transport line (quadrupole FODO array) at the exit of the Fermilab/NICADD photoinjector. It provided focusing that was sufficiently strong to contain the beam, and the transport length was sufficiently long to obtain unambiguous results from the measure. The same initial distribution of particles was then integrated with the conventional version of IMPACT-T and the new wavelet-based CPG version (with denoising turned off). Then phase-space projections computed with the two codes were compared [Bohn, et al., JDE 2, 163 (2006)]. The idea is that if the underlying dynamics of the two computed beams are similar, then the overall phase-space projections and time scales will look the same. This is the first time a test that is completely dynamical in nature has been put to use; usually comparative tests between codes are only statistical in nature, but comparisons based on dynamical evolution are far more subtle and detailed.

Figure 11 shows snapshots of the $\left(y, v_{y}\right)$-projection of transverse phase space of the same beam (same initial conditions) evolved with the conventional version (top row) and wavelet-based version (bottom row) of IMPACT-T. These are the last four frames of 'movies' of the evolution. A cross-comparison of all frames preceding these shows essentially no differences because the orbits are all regular at these earlier times. This is also true for the two panels corresponding to $t=4750-5000$ in Fig. 11, wherein the orbits are still regular (blue). Subsequently the dynamics begins to change; in the panels corresponding to $t=5000-5250$, weak chaos (green) is emerging. It is important that chaos emerges in the same frame in both simulations. Later, in the frames $t=5250-5500$, more weak chaos emerges, and now we see slight differences between the two computations. Later, in the frames $t=5500-5750$, strong chaos (red) emerges. Again, the strong chaos shows up in the same frame in both simulations, and the phase spaces differ only slightly. This is a clear indication that these two versions of IMPACT-T are in close 
detailed agreement; the underlying Poisson solvers and particle pushers yield the same dynamics. It would have been surprising (and unnerving) to see major differences because, since denoising was turned off, the level of numerical noise (PIC and macroparticle noise) is the same for both codes. Thus, we have compared 'apples to apples' and found essentially the same 'applesauce' is produced by the two distinctly different codes.

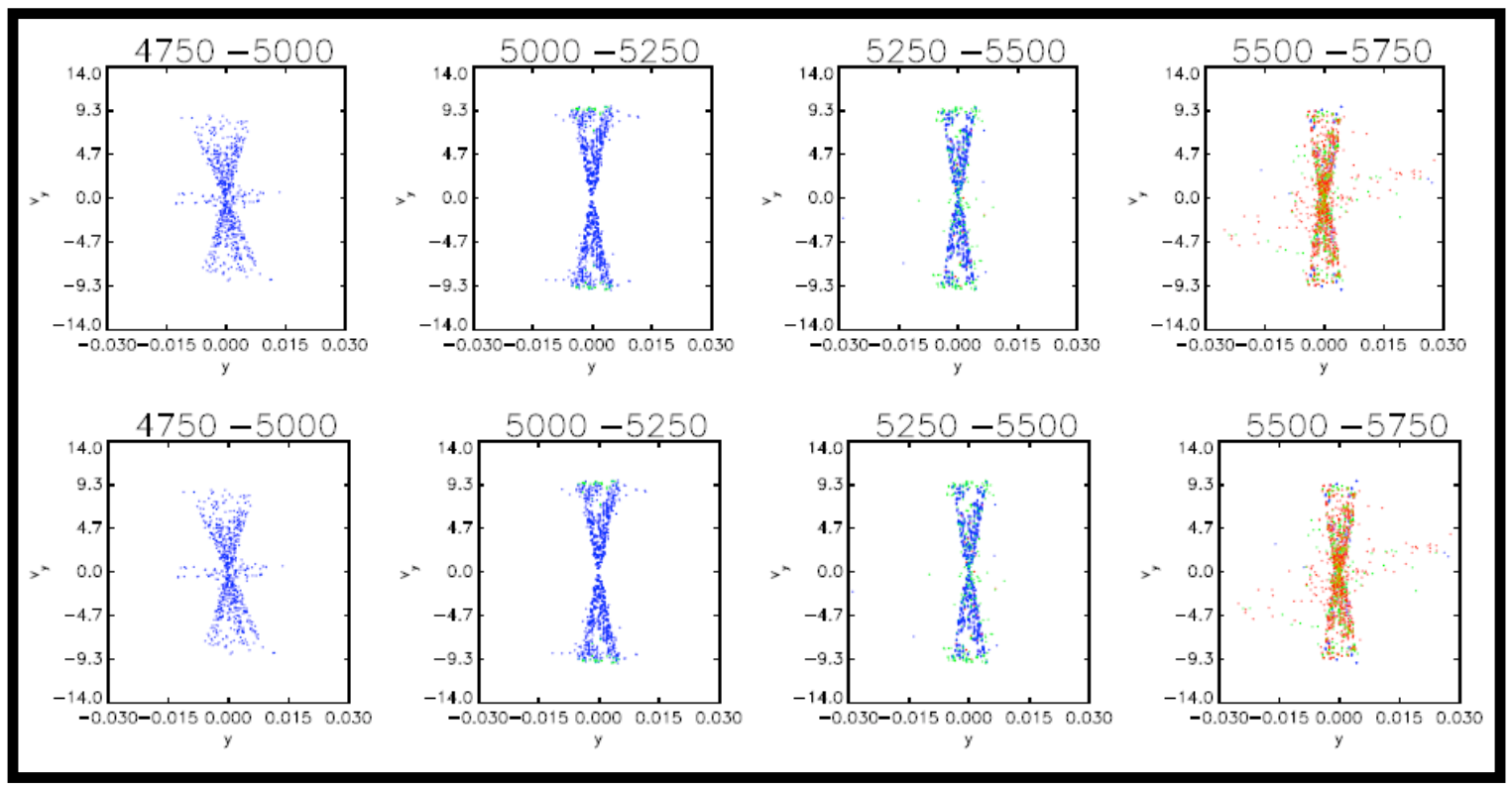

Figure 11. Phase-space projections of the evolution of the same initial electron distribution computed with the conventional version (top) and wavelet version (bottom) of IMPACT-T. Blue denotes particles on regular orbits, green denotes weakly chaotic orbits, and red denotes strongly chaotic orbits.

To summarize, the principal advantages of IMPACT-T with the wavelet-based Poisson solver are: (1) denoising, which likely yields simulation results comparable to those of a conventional code employing considerably more macroparticles; (2) compact storage and retrieval of the density and potential, a feature that will enable future comprehensive studies of halo formation and beam self-interaction via coherent synchrotron radiation (CSR); and (3) a slightly faster CPU time (by 10\%) compared to the conventional serial version of IMPACT-T. We are in the process of parallelizing and optimizing the wavelet code. Parallelizing is of most benefit for the parts of the code that do particle management, and these lie outside the Poisson solver. At this writing, we have found that parallelization yields no significant difference in run time if 100k macroparticles are used, but if $1 \mathrm{M}$ macroparticles are used, the run time is only twice that of the $100 \mathrm{k}$ particle case. This, of course, is not true for a serial code.

The code comparisons (verifications) presented above are with respect to a 'short' photoinjector. We anticipate that noticeable differences would arise between code predictions respecting long accelerators. Nonetheless, our code validations are encouraging, as are our verifications against experimental results. 
In view of these successful comparisons, one may be led to wonder whether the choice of code makes any real difference, e.g., why would IMPACT-T with 200,000 macroparticles be any better than PARMELA with 20,000 macroparticles? Perhaps, if all one seeks to control are beam moments in photoinjectors, any of these codes may suffice. However, regarding high-brightness, high-average-current beams, details matter. One example is beam halo and the dynamics of its formation. To do a reliable halo study, one needs a huge number of particles to get good halo statistics. Being able to store and retrieve the bulk potential efficiently, an ability enabled only by the wavelet-based Poisson solver, does permit such halo studies. One can populate the previously computed bulk potential with an enormous number of test charges, charges that do not contribute to the potential but respond to it, and integrate their orbits efficiently. The reason this can be done is that, because the bulk potential is now known, there is no need for further Poisson solves; the problem reduces to particle tracking. The same argument applies to the study of CSR, where now one must integrate over the history of the density to compute the retarded 4potential. Again, having the density stored compactly at each time step enables this process. These considerations perhaps constitute the compelling advantage of the wavelet-based Poisson solver.

5. Ascertain to what extent localized density enhancements that will arise from the redistribution of free energy from large scales (e.g., breathing modes) down to small scales (i.e., a hierarchy of scales) might influence the macroscopic evolution of a nonequilibrium beam.

This is inherent to applications of the newly developed wavelet code, such as the one described under Task 4 above. The degree of wavelet denoising can be adjusted to admit or screen out localized density structure, whichever is desired. Of course, one never wants to suppress real structure, and that is what motivated our careful investigation of denoising described above.

6. Ascertain the influence of unavoidable noise, e.g., that arising from machine imperfections which self-consistently drive space-charge fluctuations, on beam evolution. How do they affect the evolution of bulk properties of the beam? How do they affect the structure and extent of beam halo?

We investigated how collective space-charge modes and colored noise conspire to produce a beam halo and subsequent beam loss in Fermilab's Booster synchrotron. We showed not only that colored noise acting for a short time on a beam with strong space charge, as in a proton linac for, e.g., driving an intense source of spallation neutrons, can cause serious emittance degradation and halo growth, but also this is true if the noise acts on a beam with weak space charge for a long time, as in a synchrotron or storage ring [Bohn, AIP Conf. Proc. 737, 456 (2004)]. Consequently, a University of Rochester Ph.D. student at Fermilab, Mr. Phil Yoon, modified the code ORBIT to simulate the Booster with jitter in the magnet power supplies, the degree of which has been measured, this being done under the guidance of $\mathrm{C}$. Bohn and W. Chou. He found that the presence of noise would indeed lead to continuous emittance growth and beam loss [Yoon, et al., PAC'05, 1458 (2005)]. The cumulative beam loss after every turn is plotted in Fig. 12; after 1,000 turns, nearly $2 \%$ of the beam has impinged somewhere along the machine. 
What is more, the emittance continually grows; the synergistic interaction between colored noise arising from machine imperfections (especially jitter in the magnet power supplies) with the time-dependent space-charge force causes the root-mean-square transverse emittance to grow by about 50\%. Mr. Yoon plans to defend his dissertation in spring 2007.

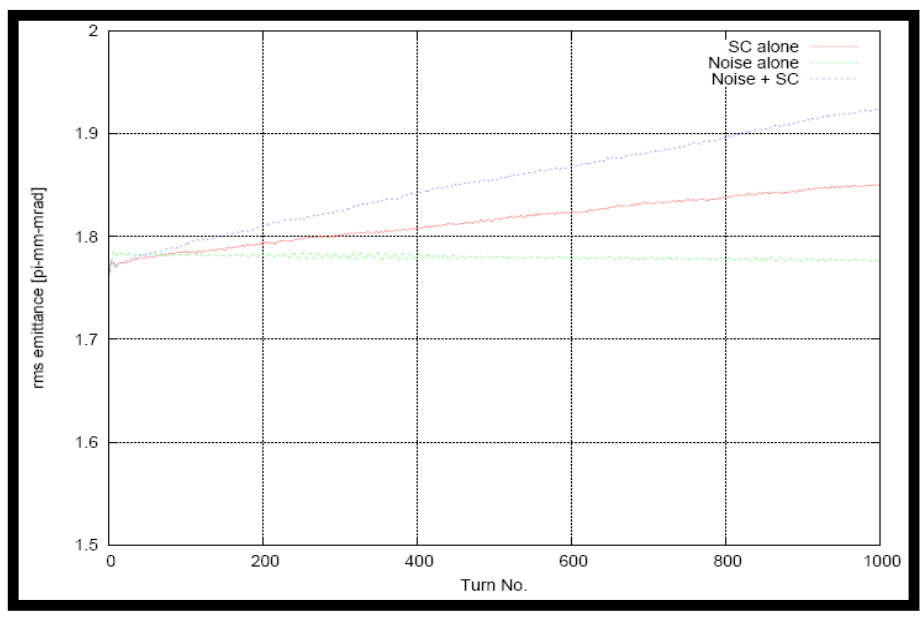

Figure 12. Emittance growth computed over 1,000 turns of the Fermilab Booster Synchrotron with realistic noise: (green) only noise is included; (red) only space charge is included; (blue) noise and space charge are included, which is representative of the real situation. Note that noise alone is not a problem, but it has a big effect on beam degradation when coupled to space charge.

Another ongoing study concerns the influence of noise in fixed-field alternating-gradient (FFAG) synchrotrons. Our collaborators are Giuliano Franchetti of GSI, Darmstadt, Germany and S. Y. Lee of the University of Indiana. The basic idea is to employ a reasonable model of the space-charge potential, combine it with an appropriate transport system, and with regard to the dipole magnets, include a kick at every turn due to sextupolar error.

The initial epoch in the evolution of an example orbit in a hypothetical one-dimensional ( $x$-only) FFAG potential is depicted in Fig. 13. Shown are the trajectory $x(t)$ (top left) and the Poincare section (top right) for that orbit. Two noteworthy features present themselves. First, by drawing a vertical line at any time $t$ in the plot of $x(t)$, one can read off the order of the resonance to which the orbit is subject at that time. Second, from both the trajectory and Poincaré plot, we see that this orbit wanders into and out of resonances, and thereby intermittently reaches higher amplitudes. This is just the initial epoch; the orbit is complicated and wanders into and out of various resonances as time advances. As a consequence, the beam itself, which is but a collection of many orbits, evolves and grows tails. This evolution is also apparent from Fig. 13 (bottom), wherein the distribution function of particles in the phase space of a single particle is plotted at various time steps.

We are now working on a similar analysis for a two-dimensional $(x, y)$ FFAG potential. It will also be of interest to explore 'dynamic noise' due to jitter in the dipole-magnet power supplies. 


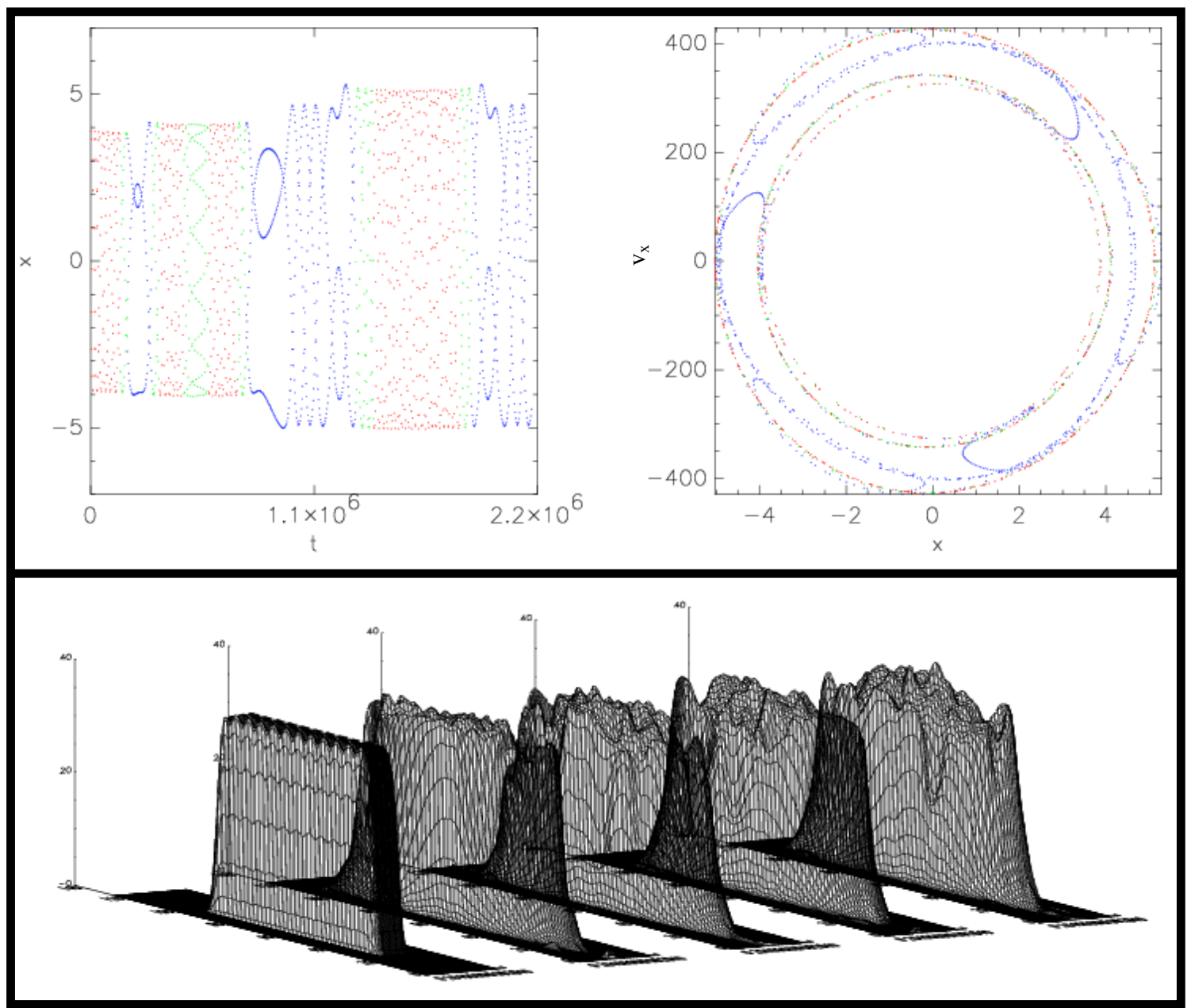

Figure 13. Trajectory $\mathrm{x}(\mathrm{t}) \mathrm{vs}$. $\mathrm{t}$ (top left) and Poincaré section (top right) of an example orbit in a 1D FFAG synchrotron potential. The distribution function of the beam (bottom) is plotted at various time steps, earlier time steps at left, and later time steps at right.

7. In close collaboration with the UMER group, design experiments to explore the physics and time scales of phase mixing and halo formation, and conduct these experiments at UMER. Correlate the results with the ongoing theoretical work. 
The University of Maryland Electron Ring (Figure 14) accelerates electrons to $10 \mathrm{KeV}$ energy, before circulating them in a ring that is approximately 3.8 meters in diameter. This is done to study how an electron's tendency to repel other electrons affects the way the beam behaves; this space charge is not noticeable at higher energies in electron beams since at a certain point the magnetic fields produced by the electrons begin to cancel out most of the natural repulsion of the charges. Space charge is a problem in machines that accelerate heavy particles such as protons since, due to their increased mass, they do not reach energies in which magnetic forces counteract space charge as quickly as electron machines do. Thus, the University of Maryland Electron Ring allows the simulation of heavy particle accelerators using a significantly smaller and cheaper set of equipment.

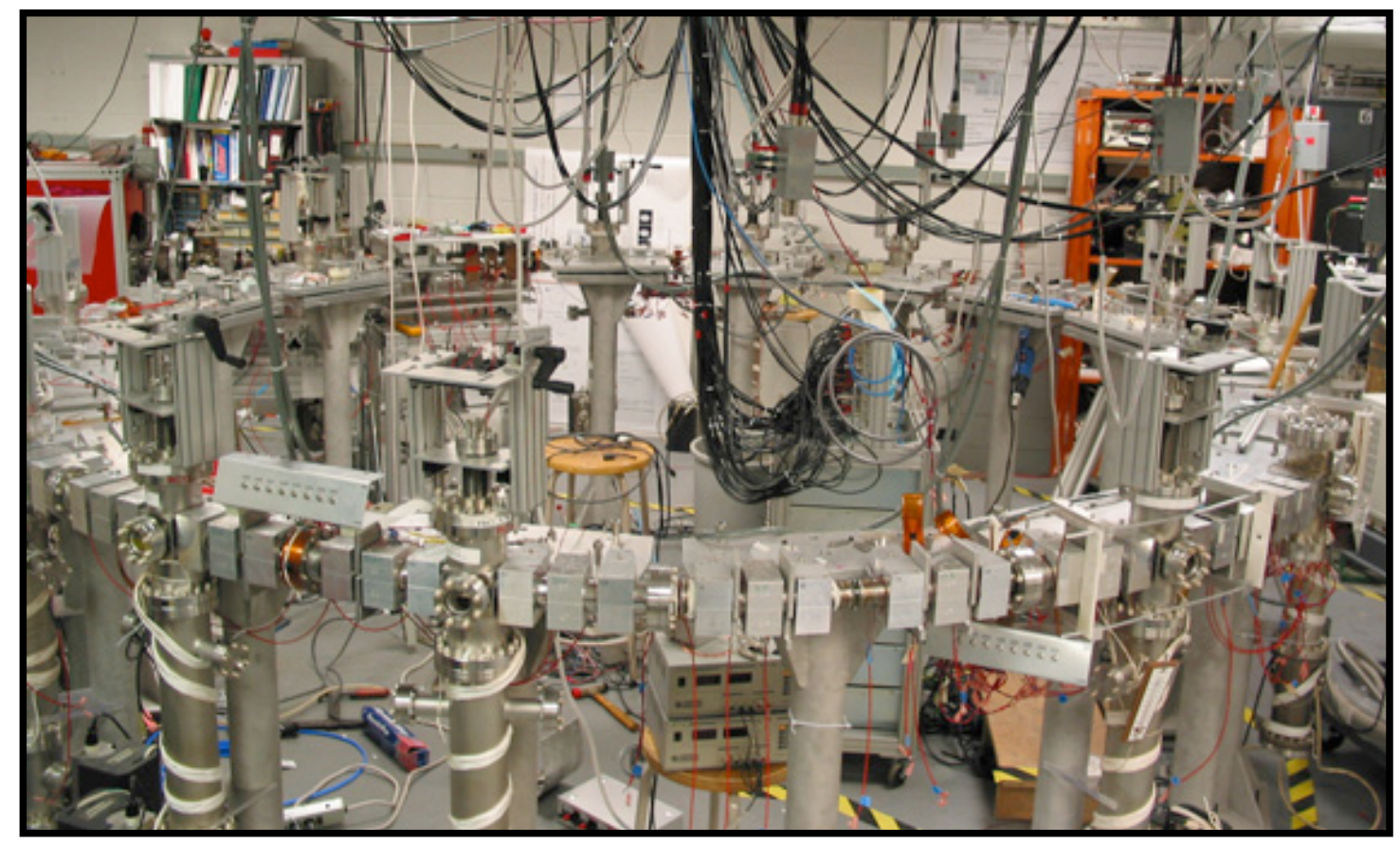

Figure 14. Picture of the University of Maryland Electron Ring (photo courtesy of www.umer.umd.edu).

In December of 2006 the Advisory Panel for the University of Maryland Electron Ring (UMER) recommended, among other things, that additional beam simulation codes be used to model the UMER. Our research built on this recommendation as well as the general mission of our group in basic beam physics and fundamental nonlinear dynamics especially as applied to accelerators and other charged particle beam devices.

The task of UMER studies is split in two parts. First, we model the ring and study the single-particle effects. We strive to optimize the ring settings that later will be tested in experiments. Once that task has been completed, a multi-particle code will be used to determine the effects of the particles in the beam on each other. We are currently pursuing the first part of the research plan, modeling the single particle dynamics of the beam line. To do this, we are using the code COSY Infinity 9.0. COSY allows for the simulation of particle beams to arbitrary order and energy. It accomplishes this using the Differential Algebraic method, which allows the numerical computation of derivatives to 
arbitrary order with machine accuracy. Furthermore, the COSY interface allows for alterations to the source code which is useful when novel machine elements need to be modeled. We emphasize that at the nonrelativistic energies of UMER and the misalignments that are implemented on purpose in the injection section of the ring one needs to be very careful about the accuracy of the usual approximations made in high energy physics. COSY Infinity is able to handle the correct beam dynamics and it proved essential for high-fidelity simulations of low current operation of the ring. This low current limit is clearly an upper bound at any current, so the optimization of the singleparticle regime will provide useful in later stages of the research in the space charge dominated regime.

Moreover, being as small and low energy as it is, the University of Maryland Electron Ring is subject to forces and effects that most beam designers can safely ignore. One of the main issues is the earth's magnetic field. Even though the earth's field is small, a low energy beam, with consequently low energy optics is going to feel the effect more than a much faster beam. Accurately taking this effect into account is a challenge, since COSY operates by calculating maps, and not directly integrating forces. Modeling the magnetic field has been done utilizing the Baker-Campbell-Hausdorf $(\mathrm{BCH})$ theorem which uses kicks applied to the coordinates of the particle in between maps created of halves of the various elements. In order to have accurate data concerning the strength of the magnetic field measurements must be taken. Those measurements provided by the University of Maryland team give data for all three axes measured at the center of each dipole, since the beam feels the earth's field in places other than the centers of the dipoles, a method of determining the field at those intermediate places had to be developed. This was accomplished by interpolating the field between the various elements using coordinate systems that were changeable from straight line coordinates within the quadrupoles and drifts to curved coordinate systems for the dipoles themselves. This allows us to have an informative view of how the earth's magnetic field can cause control issues in the beam; see Figure 15.
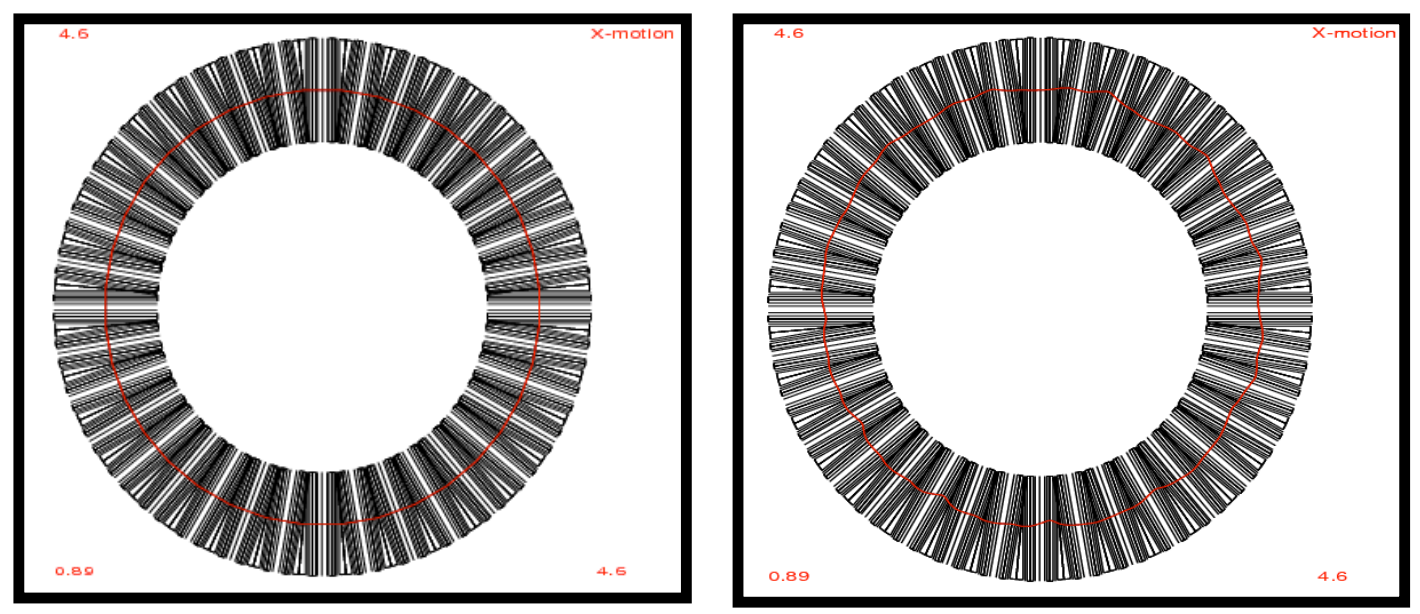

Figure 15. The left diagram shows the ring and closed orbit without the Earth's magnetic field, the right diagram shows the beam path with the Earth's magnetic field. The radial dimension has been enlarged for illustrative purposes. 


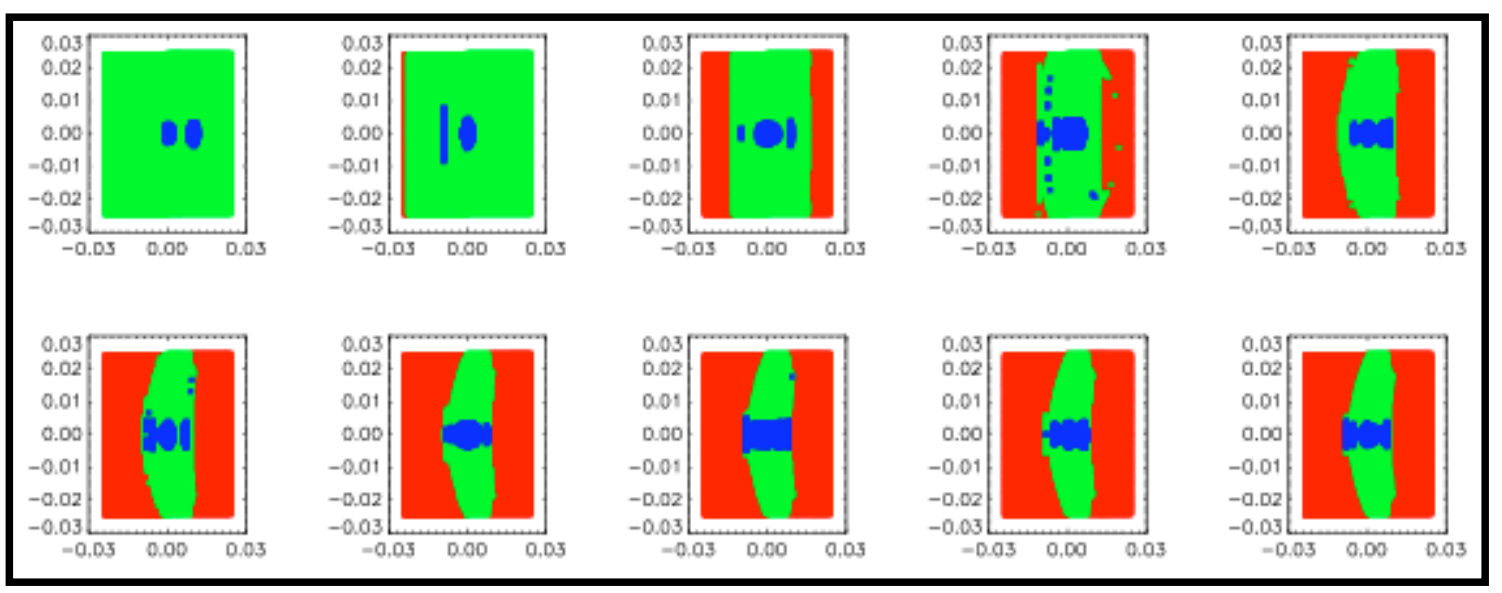

Figure 16. The evolution of the dynamic aperture (DA) through ten turns around the accelerator. After ten turns, the DA stabilizes at least up to 1000 turns. The red areas are initial conditions that have gone to infinity. The green regions are initial conditions that have remained finite, and the blue areas are initial conditions that remain within a radius of $5 \mathrm{~mm}$.

After the theoretical modeling further complications have been encountered. The beam must be placed into the ring somehow, and that can create problems due to the injection optics. The physical dislocation of the beam pipe adds asymmetries to the beam that make it difficult to run through multiple turns.

Multiple methods are being tried to determine how the part of the ring that handles the injection can be controlled. While some solutions for ring settings have been determined most have other problems that preclude their use. These problems range from solutions that are not stable, which are not useful for multi-particle beams, to solutions with magnetic fields settings that are too high. Work in progress will solve the issue by optimization that will localize the perturbation of the closed orbit to the region around the injection section and tune scans to find the optimal working point.

Since this has become such an issue current work involves studying the ring's dynamic aperture in detail. The dynamic aperture is the area within the beam pipe through which the particles that pass through stay within the beam and continue on with it, those outside tend to fly away to infinity; see Figure 16. These studies are suggesting that the dynamic aperture may be smaller that originally planned, figuring out why that is and how to remedy it is what is currently being pursued.

\section{Explore the utility of multiresolution techniques as a methodology for incorporating lessons learned about the dynamical consequences of microstructure in the beam.}

As previously mentioned, we have successfully built, implemented, and benchmarked a Poisson solver based on wavelet mathematics. As one example of our code-validation activities, not discussed above, consider the following investigation. A way to reduce space charge and consequently lower the emittance is to stretch the electron bunches longitudinally. This can be done by illuminating the photocathode with a longer laser 
pulse. We did the corresponding experiment using the Fermilab/NICADD photoinjector. We used a pulse stacker (a controllable optical delay line) to combine four successive laser pulses into a single long pulse. Stated more accurately, the long laser pulse consisted of a superposition of four shorter gaussian pulses separated by a few picoseconds (Fig. 17).

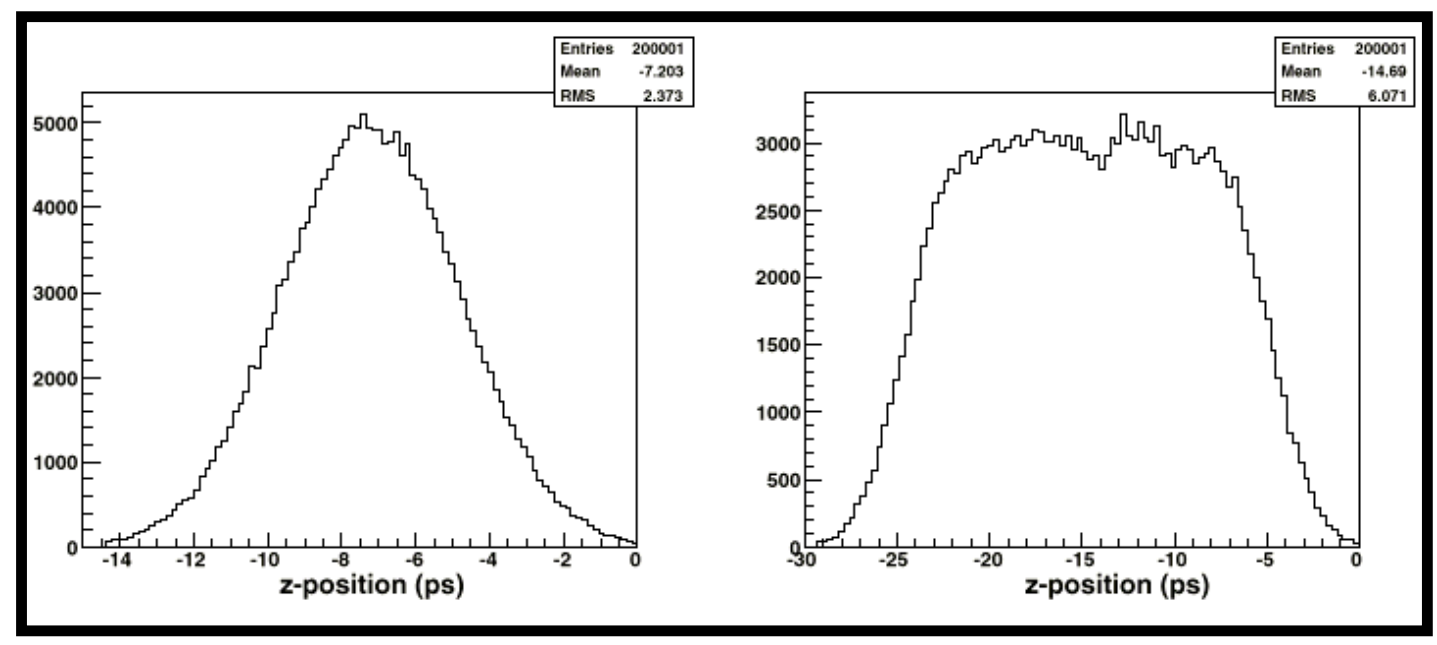

Figure 17. Single gaussian laser pulse (left). Long flat-top laser pulses (right) were obtained by stacking four single gaussian pulses separated by 5 ps.

The emittance was measured downstream of the accelerating cavities for several values of the peak magnetic field inside the gun. [Note: The peak magnetic field arises from a superposition of the magnetic fields generated by the three emittance-compensation solenoids enveloping the gun. The major contribution to this peak field comes from the primary solenoid.] Experimental results compared with simulations performed using the conventional version of IMPACT-T and the new PCG wavelet code are shown in Fig. 18(a) for laser pulses with simple gaussian shapes and in Fig. 18(b) for flat-top laser pulses. The decrease of the transverse emittance when laser pulses have a flat-top shape was much smaller than people hoped. However, agreement between the two simulation codes is quite good, and they both agree fairly well with the experimental results.

For code verification, we also have been perfecting the application of interferometry of coherent transition radiation from a beam bunch passing through a thin foil to deduce the longitudinal bunch profile [Mihalcea, et al., PRST-AB 9, 082810 (2006)]. Representing our latest results, Fig. 19 shows the interferogram and the reconstructed double-peaked longitudinal bunch profile arising from two $0.50 \pm 0.05 \mathrm{nC}$ sub-bunches that are separated by $15 \mathrm{ps}$ at the cathode after they have transited through the bunch compressor of the Fermilab/NICADD [Piot, et al., PRST-AB 9, 053501 (2006)]. The drive-laser profile at the cathode was gaussian. The phase of the accelerating (capture) cavity was set such that the first sub-bunch is significantly more compressed than the second subbunch. As Fig. 19 indicates, agreement between measurement and simulation is imperfect; however, the interferometer can clearly resolve details in structure at the pslevel ( $1 \mathrm{ps}$ is $0.3 \mathrm{~mm})$. 
As another example of code validation, Fig. 20 shows the reconstructed bunch shape downstream of the bunch compressor. In this case a single gaussian laser pulse at the cathode produces the bunch. Its charge is $3.2 \mathrm{nC}$, and compression is moderate. Consequently the coherent component of the transition radiation is farther in the infrared region where the interferometer is less sensitive. Therefore, the autocorrelation function is noisy, and the reconstructed bunch shape is less accurate. This is reflected in the different curves: each curve represents a different completion of the intensity spectrum $\left(\omega_{o} \equiv c / \sigma_{z}\right)$ for use in a Kronig-Kramers analysis. By comparison, when electron bunches are at maximum compression, the intensity of the coherent transition radiation is high and the autocorrelation function is less noisy and symmetric.
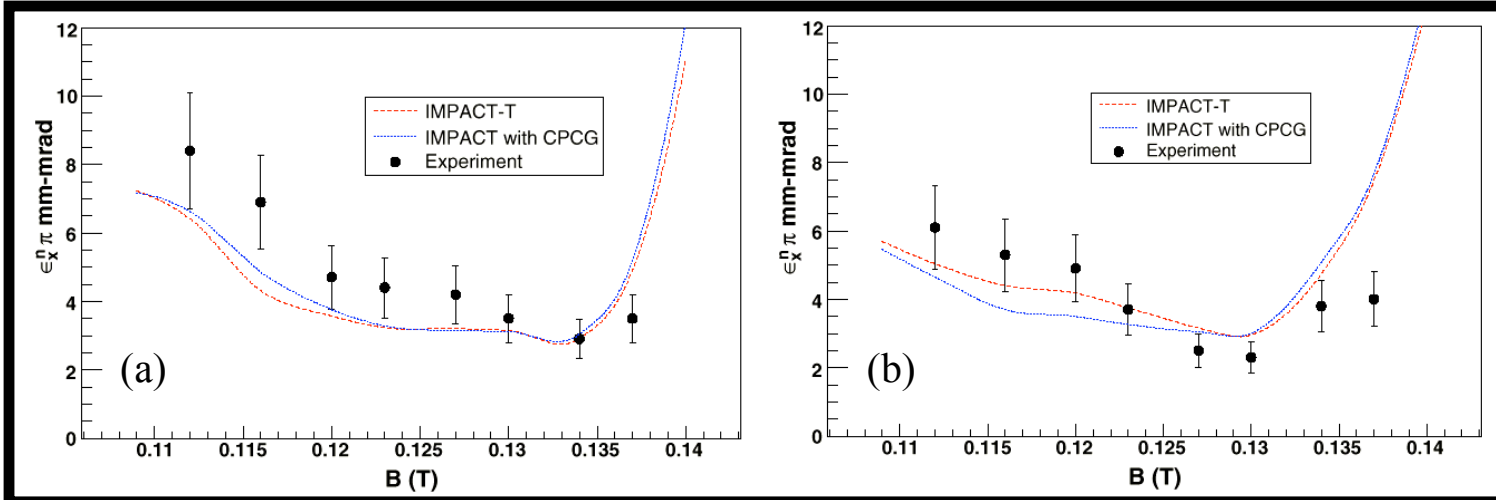

Figure 18. Experimental and simulation results for normalized transverse emittance vs. peak magnetic field inside the gun for (a) gaussian laser pulses and (b) stacked, flat-top laser pulses.

9. Apply lessons learned to specific dynamical problems concerning the production of high-brightness electron beams. These may include, but are not limited to, phasespace transformations of beams with vortex distributions (angular momentum), such as would be done in the TESLA damping rings, and bunch compression (including coherent synchrotron radiation).

As previously indicated, a key advantage of the use of wavelets is 'compression': with wavelets one can represent a density profile or potential, even if complicated, by a small number of wavelet coefficients, where 'small' is with respect to the number of macroparticles or grid points in a $N$-body PIC simulation. This means one can compute and store compactly the entire time-dependent density-potential pair computed in the course of a real machine simulation. For example, we found that for photoinjectors, one needs to retain just a few percent of the wavelet coefficients. The payoff comes in solving problems that require integrating over the history (evolution) of the beam. 


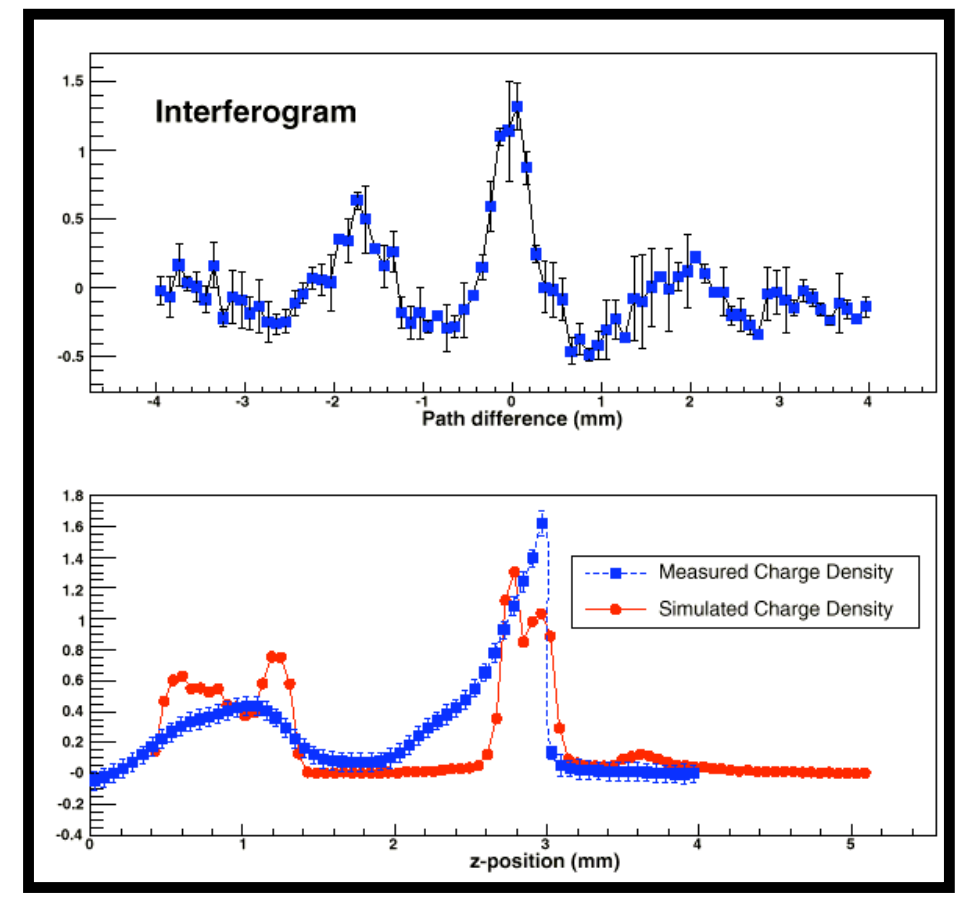

Figure 19. Autocorrelation function (top) for electron bunches consisting of two pulses separated by $15 \mathrm{ps}$ before bunch compression. Each pulse had $\sim 0.5 \mathrm{nC}$ charge. Maximum compression was set to occur at a position located between the two pulses and closer to the leading one. Longitudinal charge distributions (bottom) from experiment (blue) and Parmela simulation with 20,000 macroparticles (red). For these photoinjector settings, the overall compression factor is about 2.5 .

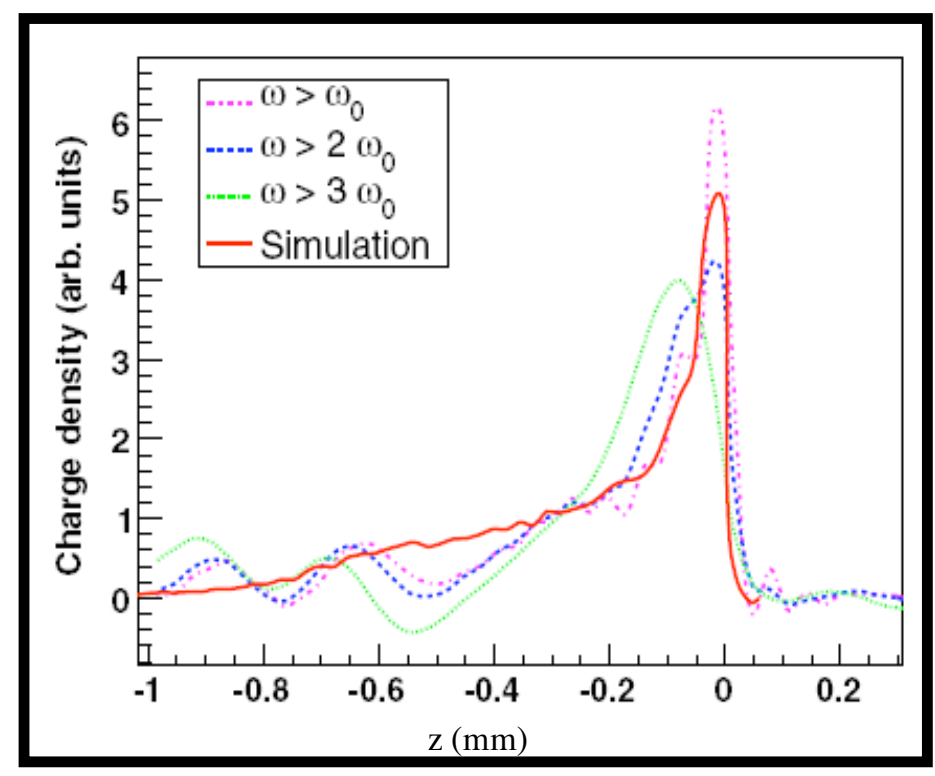

Figure 20. Variations of the longitudinal charge distribution due to intensity spectrum-completion procedure at high frequencies. The intensity spectrum at large frequencies was completed by fitting the asymptotic power function with data points from different frequency ranges. The best agreement with simulations is achieved for $\omega>2 \omega_{0}$. Experimental and simulation results were obtained for $3.2 \mathrm{nC}$ electron bunches at moderate compression. 
Two such applications are beam-halo formation and coherent synchrotron radiation (CSR). To model beam halo, one can populate the real time-dependent potential, which is compactly stored, with a large number, say $\sim 10^{6}-10^{7}$, of test particles. These particles feel the space-charge potential but contribute negligibly to it. This affords a means to compute accurately the formation and distribution of a diffuse halo, i.e., one can obtain good statistics concerning the diffuse halo. The challenge in doing so is to interpolate between the time steps for which the potential is stored; one must take care not to introduce significant numerical error when integrating the orbits. This is a task we envision attempting in the near future. One likewise can pursue fully three-dimensional computations that require the inclusion of retardation due to the finite speed of the electromagnetic field. This consideration is inherent to self-consistent interactions between wakefields and the beam. Perhaps the most important example is coherent synchrotron radiation (CSR) in magnetic bends. We envision eventually being able to do fully 3D simulations of bunch dynamics in magnetic bending systems, like bunch compressors, that include self-consistently generated CSR.

Another investigation that we recently conducted concerns the influence of a nonzero current density on bunch formation at the cathode. An illustration of the problem appears in Fig. 21. In an electron gun, the accelerating field at the cathode causes electrons in the head of the bunch to have substantially higher kinetic energy than electrons in the tail. The net effect is a substantial current density that induces a magnetic field, and this field influences the early beam dynamics. We have found that the late-time dynamics, i.e., the bunch properties downstream in a direct-current photoinjector, can be somewhat affected by this current density. This can influence how one would optimize the performance of such a machine.

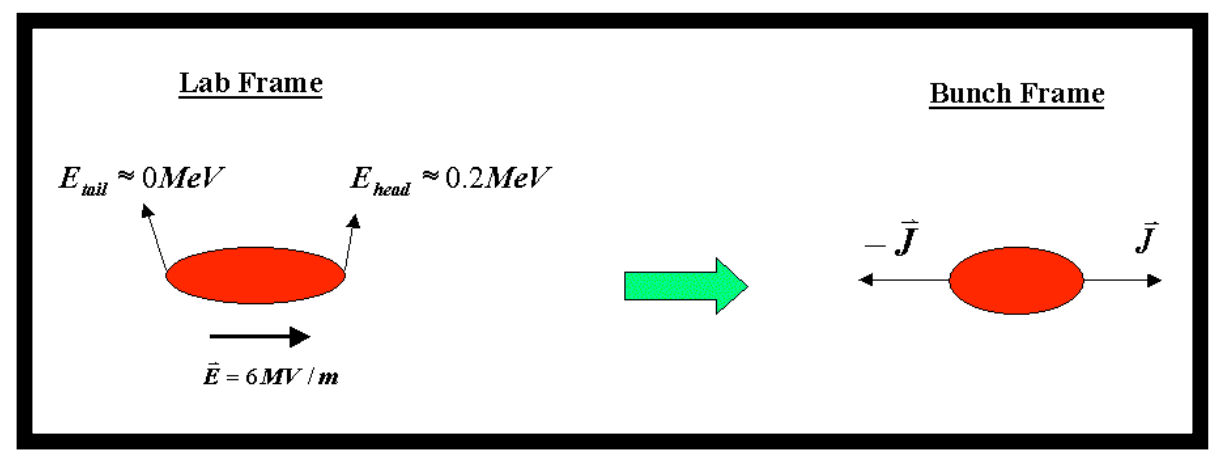

Figure 21. Generation of current density within an electron bunch due to the (direct-current) accelerating field at the cathode.

10. Explore to what extent one can use beams with space charge as laboratory analogs of large stellar systems, e.g., galaxies. What specific quantities can be measured in an evolving beam that will provide unambiguous information about the dynamics of violent relaxation in galaxies?

As discussed under Task 3 above, we now have some evidence that, in the transient stage of galactic mergers of dark-matter halos, particles are flung out by tidal shocking. Viewed most generally, this dynamics, known in the stellar-dynamics community as 
'violent relaxation', would be a facet of nonlinear Landau damping. It needs to be explored more generally; there is a danger in generalizing from a single numerical experiment! However, as indicated in the discussion of Task 1 above, we have seen an analogue of tidal shocking due to space charge in simulations of a high-brightness photoinjector. This is an example of a study that can also be done experimentally at, e.g., UMER. There, one would generate an electron beam that is far from equilibrium, perhaps a beam that involves two or more beamlets, let this beam propagate along the ring, measure its transverse properties (e.g., charge distribution, including halo), and thereby determine the time scale of the transient evolution and its effect on the charge distribution. Does it end after just a handful of betatron oscillations? Are electrons flung out due to 'tidal shocking'? This is something that can be studied both experimentally and numerically for a variety of initial conditions. The analog to transient dynamics in galaxy formation is obvious. This is a task that we eventually hope to address.

In addition, we completed a series of experiments/simulations concerning multibeamlet evolution in the AWA photoinjector. Just as would be done at UMER, a mask can be placed in front of the photocathode to generate multibeamlets. A $5 \mathrm{MeV}$ electron bunch, consisting of several beamlets separated transversely, was generated in a photoinjector and propagated in a drift space; see Fig. 22. The collective interaction of these beamlets was studied for different experimental conditions (applied external focusing strength and beamlet patterns). The experiment allowed the exploration of transverse space charge effects and compared with particle-in-cell simulations using the program IMPACT-T. Our experimental observations suggest the use of a multi-beam configuration to tailor the transverse distribution of an electron beam: we found that the central beamlet of the quincunx pattern is strongly focused by the surrounding beamlets; see Fig. 23. Our overall AWA effort provide an excellent test (validation) of simulation codes beyond standard benchmarking of moments associated to a phase space distribution. This is a critically important effort because frontier accelerators generate beams in which nonlinear dynamics, space charge, and wakefields are unavoidable. These phenomena are notoriously difficult to treat theoretically, which means code validation is essential. 


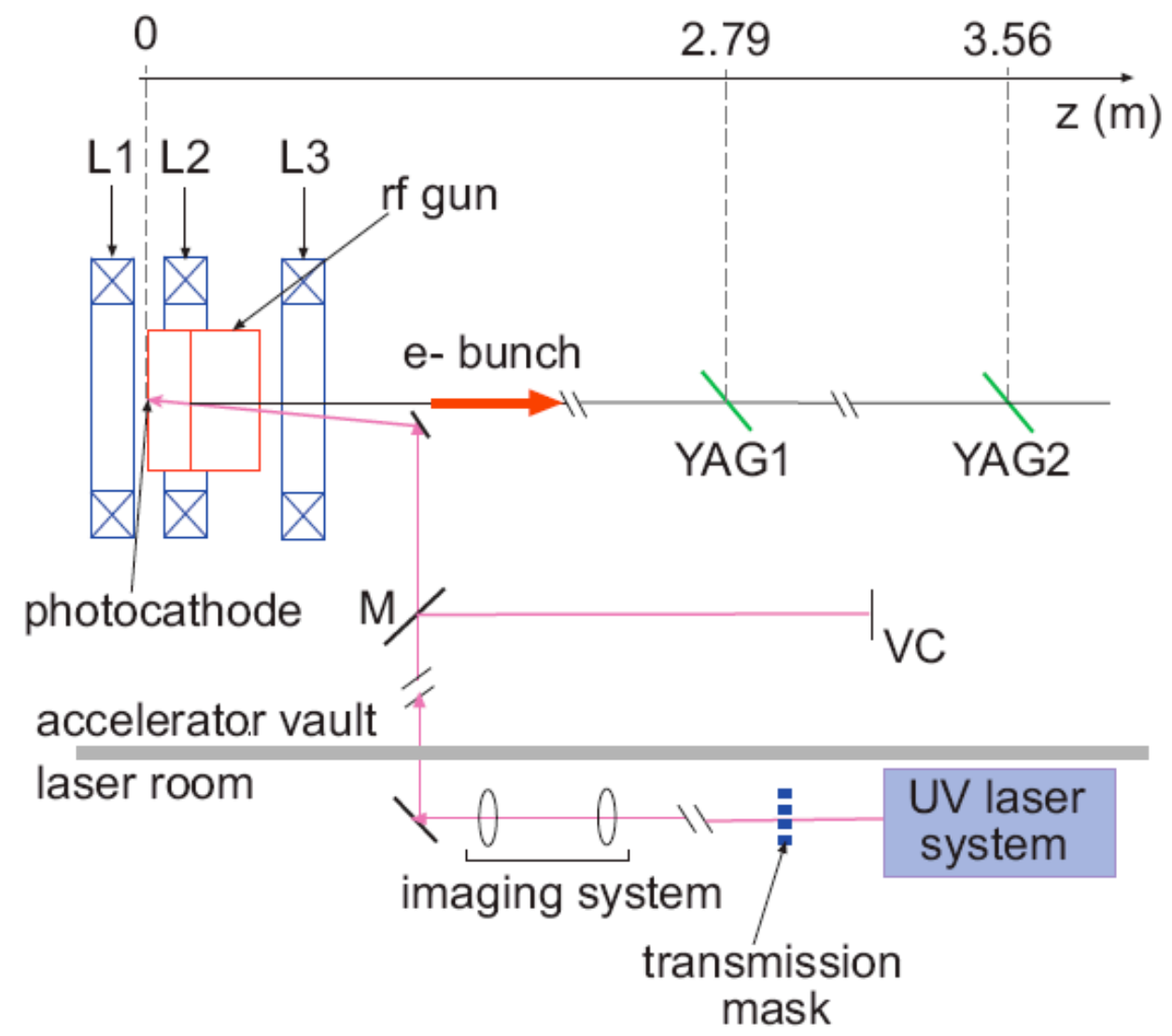

Figure 22. Overview of the AWA beam line. Here only the elements pertaining to our experiment are shown. The legend represent solenoidal magnetic lenses (L), optical mirror (M), virtual cathode (VC) and transverse density monitor (YAG1 and 2). The distance along the beamline is also shown
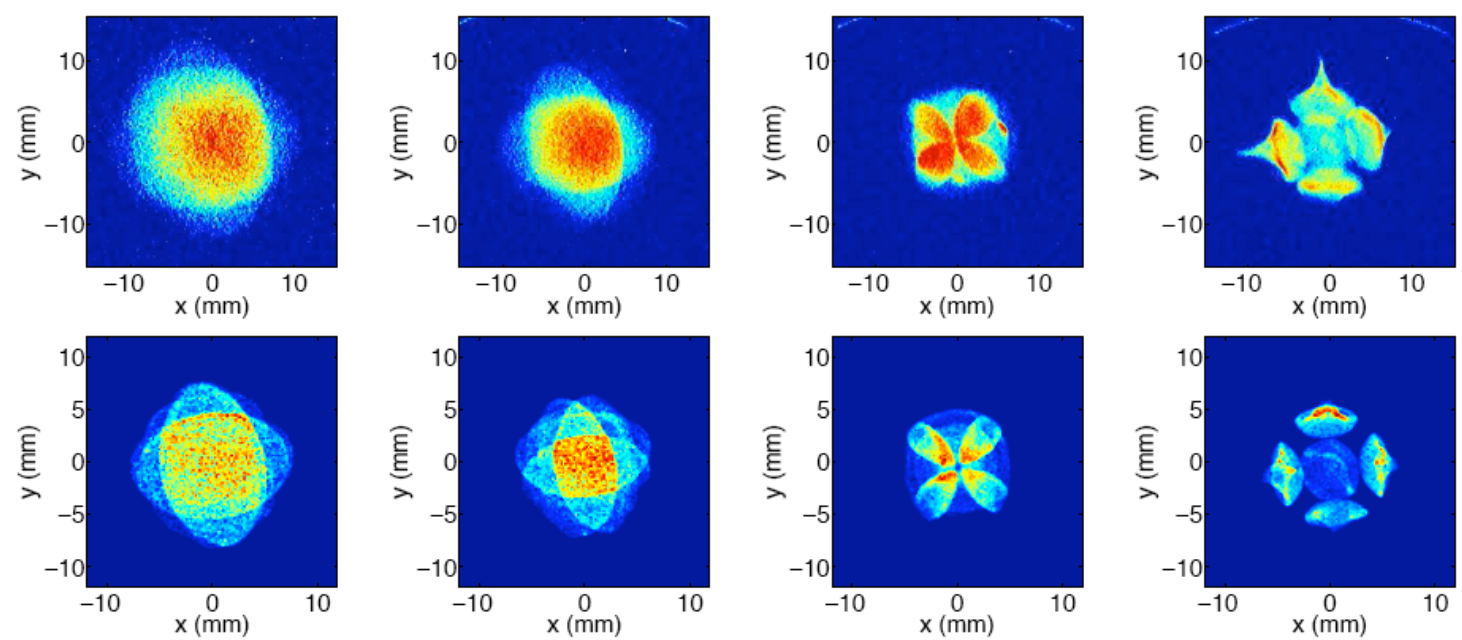

Figure 23. Propagation of a laser-generated quincunx pattern aong the AWA beamline (right four images) and comparison with IMPACT-T simulation (left four images). 


\section{Publications, Conference and Technical reports}

(For brevity, we list only refereed papers that specifically acknowledge DOE Grant No. $D E-F G 02-04 E R 41323$. To access a complete list of our many papers, go to http://www.niu.edu/nicadd/research/beam/.)

\section{Referred publications}

[1] M. Rihaoui, W. Gai, P. Piot, J. Power, Z. Yusof, "Direct observation of transverse space charge effects in a bunched electron beam", submitted

[2] B. Terzić, I. V. Pogorelov, and C. L. Bohn, "Wavelet-Based Poisson Solver for Use in Particle-in-Cell Simulations", Physical Review Special Topics - Accelerators and Beams 10: 034201 (2007).

[3] C. L. Bohn, D. Mihalcea, I. V. Pogorelov, I. V. Sideris, and B. Terzić, "Improved Simulations of Photoinjectors for High-Average-Current Free-Electron Lasers", Journal of Directed Energy 2: 163 (2006).

[4] M. Valluri, I. M. Vass, S. Kazantzidis, A. V. Kravtsov, and C. L. Bohn, "On Relaxation Processes in Collisionless Mergers", The AstrophysicalHournal 658: 741-747 (2007).

[5] D. Mihalcea, C. L. Bohn, U. Happek, and P. Piot, "Longitudinal Electron Bunch Diagnostics Using Coherent Transition Radiation", Physical Review Special Topics - Accelerators and Beams 9: 082801 (2006).

\section{Conference Proceedings}

[1] C. L. Bohn, G. T. Betzel, and I. V. Sideris, "Chaotic Dynamics and Halo Formation in Charged-Particle Beams", Nuclear Instruments and Methods of Physics Research Section A 561: 230 (2006).

[2] I. V. Sideris and C. L. Bohn, "Production of Enhanced Beam Halos via Collective Modes and Colored Noise", Physical Review Special Topics - Accelerators and Beams 7: 104202 (2004).

[3] E. Nissen and C. L. Bohn, "Influence of chaos on resonance crossing", Proceedings of the $22^{\text {nd }}$ Particle Accelerator Conference (PAC2007), Albuquerque NM, (25-29 June 2007)

[4] M. Rihaoui, C. L. Bohn, P. Piot, J. G. Power, "Impact of transverse irregularities at the photocathode on the production of high-charge electron bunches", Proceedings of the $22^{\text {nd }}$ Particle Accelerator Conference (PAC2007), Albuquerque NM, (25-29 June 2007)

[5] Y.-E Sun, J. G. Power, K.-J. Kim, P. Piot, M. M. Rihaoui, "Design study of a tranverse-to-longitudinal emittance exchange proof-of-principle", Proceedings of the $22^{\text {nd }}$ Particle Accelerator Conference (PAC2007), Albuquerque NM, (25-29 June 2007)

[6] Greg T. Betzel, Ioannis V. Sideris, Courtlandt L. Bohn, "Chaos in Time-Dependent Space-Charge Potentials", Proceedings of the 2005 Particle Accelerator Conference (PAC05), Knoxville, Tennessee (16-20 May 2005)

[7] Henry E. Kandrup, Courtlandt L. Bohn, Rami A. Kishek, Patrick. G. O'Shea, Martin Reiser, and Ioannis V. Sideris Chaotic Collisionless Relaxation in Galaxies 
and Charged-Particle Beams, Annals of the New York Academy of Sciences 1045: 12-33 (2004)

[8] Ioannis V. Sideris , "Characterization of Chaos: a New Fast and Effective Measure" Nonlinear Dynamics in Astronomy and Physics: to honor the late Professor Henry Kandrup, University of Florida, Gainesville, Florida (4-6 November 2004), Annals of the New York Academy of Sciences 1045: 79-92 (2004).

[9] C. L. Bohn, "Chaotic Dynamics in Charged-Particle Beams: Possible Analogs of Galactic Evolution", Nonlinear Dynamics in Astronomy and Physics: to honor the late Professor Henry Kandrup, University of Florida, Gainesville, Florida (4-6 November 2004), Annals of the New York Academy of Sciences 1045: 34-44 (2004).

[10] C. L. Bohn, "Collective Modes and Colored Noise as Beam-Halo Amplifiers", Proceedings of the Eleventh Advanced Accelerator Concepts Workshop, Stony Brook, New York (21-26 June 2004), American Institute of Physics Conference Proceedings 737: 456-461.

[11] John R. Cary and Courtlandt L. Bohn, "Computational Accelerator Physics Working Group Summary", Proceedings of the Eleventh Advanced Accelerator Concepts Workshop, Stony Brook, New York (21-26 June 2004), American Institute of Physics Conference Proceedings 737: 231-242.

\section{Thesis}

[1] Edward Nissen, "Chaos and its Role in Emittance Growth in Fixed Field Alternating Gradient Accelerators", August 2007

[2] Shafaq Motem, "Construction and Initial Characterization of a Low Energy Electron Source for Electron Microscopy”, August 2007

[3] Marwan Rihaoui, "Impact of Photocathode Drive Laser Transverse Density Perturbation on a High Charge Electron Beam Produced in a Photoinjector", August 2007

[4] Grigory Betzel, "Chaos in Time-Dependent Space-Charge Potentials", August 2005 